\title{
Tight Performance Bounds of Multihop Fair Access for MAC Protocols in Wireless Sensor Networks and Underwater Sensor Networks
}

\author{
Yang Xiao, Senior Member, IEEE, Miao Peng, John Gibson, \\ Geoffrey G. Xie, Ding-Zhu Du, and Athanasios V. Vasilakos, Senior Member, IEEE
}

\begin{abstract}
This paper investigates the fundamental performance limits of medium access control (MAC) protocols for particular multihop, RF-based wireless sensor networks and underwater sensor networks. A key aspect of this study is the modeling of a fairaccess criterion that requires sensors to have an equal rate of underwater frame delivery to the base station. Tight upper bounds on network utilization and tight lower bounds on the minimum time between samples are derived for fixed linear and grid topologies. The significance of these bounds is two-fold: First, they hold for any MAC protocol under both single-channel and half-duplex radios; second, they are provably tight. For underwater sensor networks, under certain conditions, we derive a tight upper bound on network utilization and demonstrate a significant fact that the utilization in networks with propagation delay is larger than that in networks with no propagation delay. The challenge of this work about underwater sensor networks lies in the fact that the propagation delay impact on underwater sensor networks is difficult to model. Finally, we explore bounds in networks with more complex topologies.
\end{abstract}

Index Terms-Under water sensor networks, upper bounds, performance evaluation, multihop.

\section{INTRODUCTION}

$\mathrm{F}$ UNDAMENTAL performance limitations must be well understood when establishing a network protocol in order to ensure that the protocol is appropriate for a particular network design choice. For example, in a bandwidth constrained system, one might rule out channelization to support the implementation of full duplex communications because they prefer to use contentionbased or coordinated-access-based protocols, even when the first option may actually be more efficient. An inappropriate protocol can result in a network which cannot sustain expected traffic loads. It is important to study the fundamental performance limitations of wireless sensor networks (WSNs), as establishing the performance bounds of a network protocol is necessary for determining whether the protocol is appropriate for a particular network design choice. The wireless sensor networks (either RF-based sensor networks or acoustic underwater sensor networks)

- Y. Xiao and M. Peng are with the Department of Computer Science, The University of Alabama, 101 Houser Hall, Box 870290, Tuscaloosa, AL 35487-0290. E-mail: yangxiao@ieee.org.

- J. Gibson is with the Department of Computer Science, Graduate School of Operational and Information Sciences, Monterey, CA 93943.

E-mail: jhgibson@nps.edu.

- G.G. Xie is with the Naval Postgraduate School, 1411 Cunningham Rd., Glasgow Hall East Wing, Monterey, CA 93943-5201.

- D.-Z. Du is with the Department of Computer Science, University of Texas at Dallas, 800 W. Campbell Road, MS EC31, Richardson, TX 75080.

E-mail: dzdu@utdallas.edu.

- A.V. Vasilakos is with the Department of Computer and Telecommunications Engineering, University of Western Macedonia, Greece.

E-mail: vasilako@ath.forthnet.gr.

Manuscript received 6 Nov. 2009; revised 1 June 2011; accepted 12 Aug. 2011; published online 25 Aug. 2011.

For information on obtaining reprints of this article, please send e-mail to: tmc@computer.org, and reference IEEECS Log Number TMC-2009-11-0487. Digital Object Identifier no. 10.1109/TMC.2011.190. considered in this paper are multihop: each sensor node performs sensing, transmission, and relay. All data frames are sent to a dedicated data-collection node, called the base station, that is responsible for relaying the frames to a dislocated command center over a radio or wired link.

For this study, we first consider the linear network, a commonly used topology designed by researchers from UC Santa Barbara for moored oceanographic applications [1], in which an array of equally spaced underwater marine sensors is suspended from a mooring buoy. All data in the network flow to a base station above the water's surface which is responsible for storing and relaying all collected data to a command center via an aerial radio link. During an event of interest, (e.g., a storm), it is desirable for the command center to acquire near real-time readings from all of the sensors in order to calibrate them as the event progresses [1]. An equally appropriate employment would include a collection of seismic sensors, perhaps a long grid topology, along a potential tsunami path that would monitor the movement of the wave phenomena over a relatively short distance and relay the collected data samples through the base station to an observatory station, as the radio signal would travel nearly 200,000 times faster than an acoustic signal. For such real-world applicable networks, it is critical that the medium access control (MAC) protocol [6], [7], [8], [9], [10], [11] ensure that each sensor has an equal opportunity to forward its local observations to the command system in order to establish trends or to detect anomalies.

In this paper, we introduce the notion of fairness for sensor data delivery to this environment and support the application of a fair-access criterion to the MAC protocols under consideration for use in both RF-based WSNs and underwater acoustic sensor networks (UASNs). Employing 
a fair-access MAC protocol, however, may have a negative impact on the network's performance in terms of reduced throughput of data delivery to the base station and increased average frame latency, as those stations furthest from the base station must compete with nodes closer to the base station for the limited network capacity, while those closer to the base station incur a greater traffic load as they must relay all traffic received from the upstream (predecessor) nodes. This paper analyzes such an impact by deriving tight bounds on the network utilization and frame latency performance of fair-access MAC protocols for linear topology and for two-row grid topologies. The bounds are significant because they hold for any MAC protocol conforming to the fair-access criterion, such as contentionbased protocols (e.g., Aloha or CSMA based) or contentionfree protocols (TDMA, etc.) under both single-channel and half-duplex radios. We show that these bounds are tight by proving that they can be achieved by a particular TDMA scheduling algorithm. We also show how to obtain the performance bounds of more complex grid topologies using the analysis method employing by linear topology and tworow grid topologies.

The existence of a computationally traceable optimal fairaccess protocol is interesting because it has been shown that the general problem of optimal scheduling for a multihop network is NP-complete [2]. This may be because we consider only the topology in which the routing structure is simple. The data forwarding paths of a linear or grid network can be modeled as a tree. While tree-based scheduling may be too restrictive for arbitrary ad hoc networks [3], such an approach seems appropriate for networks in which all traffic must flow to a collective base station, which essentially forms a root node. The flow of traffic along the branches of the tree must be deconflicted with the flow of traffic along other branches so that collisions or interference between branches is eliminated or minimized. Individual node transmission windows may be adaptive [4] or static, as described herein. While a multihop star topology may be of particular interest, a linear one is directly applicable to buoyed networks. Furthermore, if the branches of the star are noninterfering, then it is the final hop of the star by which each branch connects to the base station that must be carefully controlled in order to limit collisions.

We also examined the effect of the end-to-end performance bounds on the traffic generation rate and the sensing interval of individual sensors. This paper presents an analysis that confirms that the maximum feasible load offered by each sensor node is inversely proportional to the size of the network, which implies that multiple smaller networks may be inherently preferable to fewer larger networks.

In short, the contributions of this paper are given as following. First, this paper presents the concept of fair access, which applies to both WSNs and UASNs. We then present a formal analysis of the utilization and delay bounds of specific linear or grid networks that require fair access. Next, we provide analysis of bounds in more complex topologies. The significance of these bounds is two-fold: First, they are universal (i.e., they hold for any MAC protocol) under both single-channel and half-duplex radios; second, they are provably tight (i.e., they can be achieved by a version of the time division multiple access (TDMA) protocol that is selfclocking and therefore does not require system-wide clock synchronization). In addition, this formal analysis provides a feasible way to estimate the performance bounds of more complex topologies. Therefore, in Section 8, we present the analysis results for general $k \times n$ grid network. Finally, the performance bounds of underwater sensor networks are explored with the consideration of propagation delays. A tight upper bound on network utilization is derived for the case in which propagation delay is less than or equal to half of the frame transmission time, which demonstrates that the utilization in networks with propagation delays is larger than in networks without propagation delays. The challenge lies in the fact that the propagation delay impact on underwater sensor networks is difficult to model.

The rest of this paper is organized as follows: Section 2 reviews the related work. Section 3 provides a problem formulation; Section 4 studies RF-based WSNs; Section 5 studies UASNs; Sections 6 and 7 provide performance analysis for WSNs and USANs, respectively; Section 8 presents analysis of bounds in more complex topologies; Section 9 provides the simulation results; finally, we conclude this paper in Section 10.

\section{Related Work}

In many applications of sensor networks, data frames generated by every node need to reach the base station. In this scenario, the communication pattern is many-to-one also known as convergecast [12], [13]. Convergecast can be accomplished by employing either contention-based MAC protocols like CSMA or contention-free MAC protocols like TDMA. Contention-based MAC protocols usually consume more energy than TDMA protocols since they waste energy during collisions and idle listening [14]. For example, a traffic monitoring network using the TDMA protocol described in [15] has a lifetime of 1,000 days, compared to 10 days for a network using contention-based MAC protocols. Thus, many applications in sensor networks employ TDMA scheduling algorithms [2], [3]. These algorithms aim to minimize the number of time slots required for each node to communicate once with all its neighbors. However, these algorithms might incur high latency in the Convergecast scenario.

The authors in [16], [17] proposed algorithms to obtain the minimum delays in collecting sensor data for networks of various topologies such as line, multiline, and tree. In these papers, they approached the problems from the way that base station sends frames to the sensor nodes. In addition, the algorithms proposed in [16], [17] are centralized such that the schedule is computed at the base station and requires cooperation between nodes. However, these requirements may not be practical in some sensor network applications. The authors in [12] proposed a distributed minimal time convergecast scheduling process in which each node computes its own schedule after the initialization phase. However, all of the scheduling algorithms for multiline topology networks in [12], [16], [17] assume that there is no interference between different routing routes. Furthermore, tree networks can be reduced into equivalent multiline networks, as in [12], [16], [17], and thus the 
proposed algorithms for the tree topology also have implicit assumptions above.

As in [12], the optimal TDMA fair scheduling in our paper is also distributed. Although the scheduling algorithm for linear topology in our paper is similar to the line case in [12], we propose a novel method to place lower bounds on data collection times. Unlike in the previous work, the multiline topology networks referred to as grid topologies in our paper assume that there exists interference between different routing routes; our scheduling algorithms for a grid topology are therefore more complicated. In addition, all previous works were focused on terrestrial wireless sensor networks and have not considered the propagation characteristics of the underwater wireless medium. Many papers [5], [6], [7], [8], [9], [21], [22], [23], [24], [25], [26], [27] have addressed MAC in underwater sensor networks, but they have not considered our problems. This paper addresses the impact of nontrivial propagation delays, a definitive characteristic of underwater acoustic networks. Considering nontrivial propagation delays, this problem is difficult to study, as shown in the approach presented in Section 5. For example, we demonstrate that the utilization in networks with propagation delay is larger than in networks with no propagation delay under certain conditions. Another difference of this paper from the previous work is that we consider utilization under the fair-access criterion introduced in the next section.

Note that in this paper the derived upper bounds hold for any MAC protocol (including CSMA, TDMA, Aloha, etc.) under both single-channel and half-duplex radios. For more information on upper limits for CSMA-like MAC proposals such as CSMA-CA, the readers may refer to [19], [20].

Just for the illustration purpose, if we do not consider the average performance and consider only one round of transmissions, a CSMA protocol could accidentally act as an optimal TDMA protocol for a short time; therefore, the tight bound could also be achieved by CSMA protocols for that short period of time.

\section{Problem Formulation}

In this section, we first present the sensor network model; then we give the fair-access criterion definition, based on which we formulize the optimization problem under a few assumptions. Lastly, we describe the linear and grid topologies on which we explore the tight upper bounds on network utilization.

Sensor network definition. Consider a wireless sensor network comprised of a base station (BS) and $n$ sensor nodes, denoted as $O_{i} ; i=1,2, \ldots, n$. Sensor nodes generate sensor data frames and send them to the BS. Some sensor nodes perform the additional task of forwarding/routing frames to the BS, (i.e., a frame may need to be relayed by several nodes in order to reach the BS).

Note that the above definition is not limited to a particular topology. Let $U(n)$ denote the utilization of the above network, (i.e., the fraction of time that the BS is busy receiving correct data frames). Let $G_{i}$ denote the contribution of (i.e., data generated by) sensor $O_{i}$ to the total

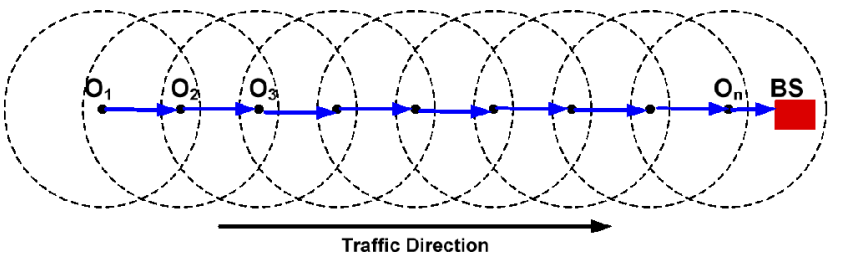

Fig. 1. A linear topology.

utilization. The following holds: $U(n)=\sum_{i=1}^{n} G_{i}$. Implicit in the utilization is the impact of propagation delays. As noted, these delays can be significant for UASNs, especially when compared to more traditional RF-based wireless networks.

Suppose that the network is required to use a MAC protocol that ensures all hosts are provided with the capability to contribute equally to the composite throughput. The impacts of such a criterion on RF-based WSNs (negligible propagation) and UASNs (nonnegligible propagation) are considered in this paper. The criterion is presented as follows:

Fair-access criterion definition. A MAC protocol used by the sensor network satisfies the fair-access criterion if all sensor nodes contribute equally to the network utilization. In other words, if the following condition holds:

$$
G_{1}=G_{2}=\cdots=G_{n} .
$$

Optimization objective and assumptions. Consider a sensor network like the one described above. The optimization problem maximizes $U(n)$ under the fair-access criterion. In the remainder of this paper, we investigate this problem under the following assumptions:

1. All data frames are of the same size.

2. All sensor nodes have the same transmission capacity.

3. Acknowledgments are either implicit via piggyback or are explicit and out-of-band.

4. In-network sensor data processing is not used.

5. If two sensor nodes are within one-hop, one sensor node's transmission will interfere with the other's reception.

6. Internal node processing delays, which are associated with frame storage and queuing within a node, are negligible. Propagation delay is negligible for WSNs, but not for USANs.

7. Other characteristics, such as variable propagation delay, frequency dependent path loss, fading, noise and Doppler spread of USANs are not discussed in this paper.

Linear topology. The topology is illustrated in Fig. 1. $n$ sensor nodes and a BS are placed in a linear fashion. Assume that the transmission range of each node is just one hop and that the interference range is less than two hops. In other words, only neighboring nodes have overlapping transmission ranges. As shown in Fig. 1, $O_{i}$ generates sensor data frames and sends the frames to $O_{i+1}$. $O_{i}$ also relays data frames received from $O_{i-1}$ to $O_{i+1}$. Finally, $O_{n}$ forwards data to the BS, which collects all of the data frames.

2-row grid topology. The 2-row grid topology is illustrated in Fig. 2. The transmission ranges are such that 

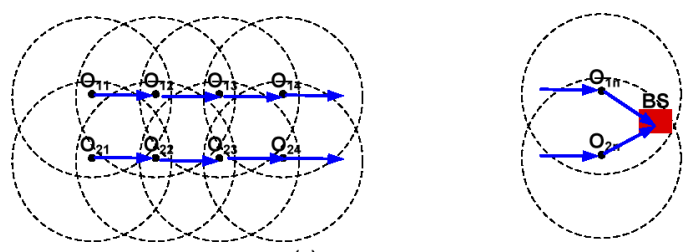

(a)
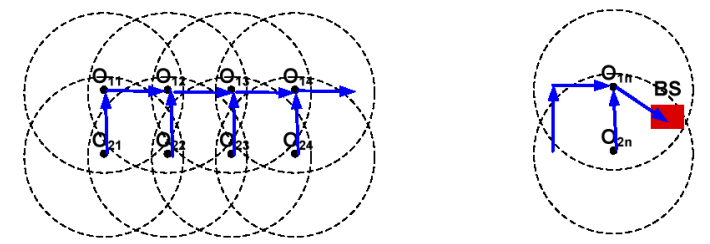

(b)

Fig. 2. Grid topology with two rows of sensors.

horizontal or vertical neighbors can hear each other but two diagonal neighbors cannot. Two different routing patterns are considered: 1) the two rows forward data frames independently, as illustrated in Fig. 2a, or 2) the bottom sensors forward data to the top row first, as illustrated in Fig. $2 \mathrm{~b}$. The results for this grid can be extended to grids with more rows, but such results are not included here due to space limitations. Some more complex topologies are introduced in Section 8.

Different routing patterns behave differently since neighboring routing paths may interfere if the routing patterns are different. The number of routing patterns is exponentially large (with all kinds of combinations). Therefore, it is both impossible and unnecessary to study all of the routing patterns. Instead, we select some representative patterns.

\section{RF-Based Wireless Sensor Network (Not UNDERWATER)}

In this section, we first derive upper bounds on network utilization for two specific topologies, linear and 2-row grid, under the fair-access criteria in RF-based wireless sensor network. Then, we show that derived upper bounds are indeed achievable by a particular TDMA scheduling algorithm.

\subsection{Derivation of Utilization and Delay Bounds}

In this section, we derive upper bounds on $U(n)$ and lower bounds on the effective intertransmission delay of a node, that is, the time between samples for a given node for two specific topologies, linear and 2-row grid, under the fairaccess criteria. We then present three theorems which establish the performance bounds. Finally, the proofs of the theorems are given for completeness.

Theorem 1. For the linear topology, under fair access, $U(n)$ is upper bounded by the optimal utilization, $U_{\text {opt }}(n)$

$$
U(n) \leq U_{\text {opt }}(n)= \begin{cases}n /[3(n-1)], & n>1, \\ 1, & n=1 .\end{cases}
$$

An asymptotic lower limit for the optimal utilization exists and is equal to $1 / 3$.

Moreover, the intersample time for each node, denoted by $D(n)$, is lower bounded by the minimum effective transmission delay for the node, or minimum cycle time, $D_{\text {opt }}(n)$

$$
D(n) \geq D_{\text {opt }}(n)= \begin{cases}3(n-1) T, & n>1 \\ T, & n=1,\end{cases}
$$

where $T$ is the transmission time of one data frame.

Theorem 2. For the 2-row grid topology with the routing pattern as illustrated in Fig. 2a, under fair access, $U(2 n)$ is upper bounded by the optimal utilization, $U_{\text {opt }}(2 n)$

$$
U(2 n) \leq U_{\text {opt }}(2 n)=2 n /(3 n-1) .
$$

The asymptotic lower limit for the optimal utilization is $\frac{2}{3}$.

Moreover, $D(2 n)$ is lower bounded by the minimum intersampling time, $D_{\text {opt }}(2 n)$

$$
D(2 n) \geq D_{\text {opt }}(2 n)=(3 n-1) T,
$$

where $T$ is the transmission time of one data frame.

Theorem 3. For the 2-row grid topology with the routing pattern depicted in Fig. $2 b$, under the fair-access criterion, $U(2 n)$ is upper bounded by the optimal utilization, $U_{\text {opt }}(2 n)$ :

$$
U(2 n) \leq U_{\text {opt }}(2 n)= \begin{cases}2 n /(6 n-5), & n \geq 2 \\ 2 / 3, & n=1 .\end{cases}
$$

The asymptotic lower limit for the optimal utilization is $1 / 3$. Moreover, $D(2 n)$ is lower bounded by the minimum transmission delay, or time between samples, $D_{\text {opt }}(2 n)$ :

$$
D(2 n) \geq D_{\text {opt }}(2 n)= \begin{cases}(6 n-5) T, & n \geq 2 \\ 3 T, & n=1,\end{cases}
$$

where $T$ is the transmission time of one data frame.

The significance of Theorems 1-3 is that they provide optimal bounds on utilization, regardless of the MAC protocol employed. In other words, no matter which MAC protocol is used, whether contention-free (TDMA, token passing, etc.) or contention-based (CSMA, aloha, etc.), the bounds hold as long as the protocol conforms to the fairaccess criterion. In order to prove optimality, we must prove that 1) the bounds hold for any fair-access conforming MAC protocol and that 2) the bounds are indeed achievable by at least one protocol.

Note that there are $n$ nodes in Fig. 1 , but $2 n$ nodes in Fig. 2, as reflected in the notation for the network utilization and the minimum intersample time, or transmission delay.

Before showing the actual proofs, let us provide some of the intuition behind them. The fair-access criterion requires that $G_{1}=G_{2}=\cdots=G_{n}$ for the linear network. Let $x$ denote the time period during which the BS successfully receives at least one original data frame from each sensor node in the network. It is clear that $x$ is a random variable, that we can derive the minimum value of $x$, and that the maximum utilization is also achieved when the minimum value of $x$ is achieved. During the time period $x$, the BS has busy time (denoted as $b$ ) receiving frames and idle time (denoted as $y$ ) when it is either blocked or waiting for its upstream neighbor to send. Thus, $x=b+y$. Note that $x$ is the cycle time for the network under the fair-access criteria and that it determines the effective transmission delay for a node with a static ordering of relayed frames. For discussion purposes, we use a frame and the time period of transmitting/ receiving a frame interchangeably in the following proofs. 
Since we assume no particular MAC protocol, frames may be lost, corrupted, or delayed due to collisions or queuing.

Proof of Theorem 1. For $\mathbf{n}>2$ : During the time period $x$, the BS needs to receive at least $n$ frames from $O_{n}$ because frames may be lost or delayed as noted above. Thus, $O_{n}$ transmits at least $n$ frames (including $n-1$ relayed frames and one of its generated frames). We therefore have $b \geq n T$. Likewise, in order for $O_{n}$ to receive $(n-1)$ frames from $O_{n-1}, O_{n}$ needs to listen to at least $(n-1)$ frames, during which time the BS must be idle. Furthermore, when $O_{n-2}$ transmits, $O_{n}$ cannot transmit since they are within two-hops (i.e., $O_{n}$ 's transmissions will interfere with the frame reception by $O_{n-1}$ from $\left.O_{n-2}\right) . O_{n-2}$ needs to transmit at least $(n-2)$ frames to $O_{n-1}$, during which time $O_{n}$ cannot transmit. Therefore, the total time in which $O_{n}$ cannot transmit is $y \geq(n-1) T+(n-2) T$. Therefore, we have

$$
x=b+y \geq n T+(n-1) T+(n-2) T .
$$

Since $D(n)=x$, we were able to derive (3) for the case of $n>2$. During the time period $x$, the BS may receive more than $n$ frames, but only $n$ frames can be counted in the utilization under the fair-access criterion. Since we must minimize $x$ to achieve the optimal utilization, we have

$$
\begin{aligned}
U(n) & =n T / x \leq n T /[n T+(n-1) T+(n-2) T] \\
& =n /[3(n-1)],
\end{aligned}
$$

which proves (2) for the case of $n>2$.

Since $\lim _{n \rightarrow \infty} n /[3(n-1)]=1 / 3,1 / 3$ is the asymptotic lower limit for the optimal utilization.

For $\mathbf{n}=2$ : Since we want $G_{1}=G_{2}$ during the time period $x, O_{2}$ transmits at least two frames (one relayed frame and its own). We have $b \geq 2 T . O_{2}$ needs to listen to at least one frame from $O_{1}$. We have $y \geq T$ and thus $x=b+y \geq 3 T$. Since $D_{\text {opt }}=x$, we were able to derive (3). Since we must minimize $x$ to achieve the optimal utilization, $U(n)=2 T / x \leq 2 T / 3 T=2 / 3$, which proves

(2) for this case.

For $\mathbf{n}=1$. Obviously, $U(1) \leq 1$ and $D(1) \geq T$.

Proof of Theorem 2. For $\mathbf{n}>2$ : Under the fair-access criterion, during the time period $x$, the BS needs to receive at least $n$ frames from $O_{1 n}$ because frames can collide, be corrupted, or be delayed (i.e., $O_{1 n}$ transmits at least $n$ frames (including $n-1$ relayed frames and one of its generated frames) to the BS). Likewise, $O_{2 n}$ transmits at least $n$ frames to the BS. We therefore have $b \geq 2 n T$. In order for $O_{1 n}$ to receive $n-1$ frames from $O_{1(n-1)}$ and for $O_{2 n}$ to receive $n-1$ frames from $O_{2(n-1)}, O_{1 n}$ and $O_{2 n}$ need to listen for at least $(n-1)$ frames. Note that when $O_{1(n-2)}$ transmits, $O_{1 n}$ cannot transmit but $O_{2 n}$ can. Similarly, when $O_{2(n-2)}$ transmits, $O_{2 n}$ cannot transmit but $O_{1 n}$ can. So, the total time in which neither $O_{1 n}$ nor $O_{2 n}$ can transmit is $y \geq(n-1) T$. Thus, we have $x=b+y \geq 2 n T+(n-1) T$. Since $D_{\text {opt }}=x$, we were able to derive (5) for this case. During the time period $x$, the BS may receive more than $2 n$ frames, but only $2 n$ frames can be counted in the utilization under the fair-access criterion. To achieve the optimal utilization, we minimize $x$, yielding

$U(2 n)=2 n T / x \leq 2 n T /[2 n T+(n-1) T]=2 n /(3 n-1)$.

The rest of the proof is omitted for brevity.

Proof of Theorem 3. For $\mathbf{n}>2$ : Under the fair-access criterion, during the time period $x$, the BS needs to receive at least $2 n$ frames from $O_{1 n}$, as shown above. We therefore have $b \geq 2 n T$. In order for $O_{1 n}$ to receive $2(n-1)$ frames from $O_{1(n-1)}$ and one frame from $O_{2 n}, O_{1 n}$ must listen for at least $2(n-1)+1$ frames. Furthermore, when either $O_{1(n-2)}$ or $O_{2(n-1)}$ transmits, $O_{1 n}$ cannot transmit. $O_{1(n-2)}$ must transmit at least $2(n-2)$ frames, and $O_{2(n-1)}$ must transmit at least one frame (if frames collide, are corrupted, or delayed more frames are needed). Thus, we have $y \geq 2(n-1) T+T+2(n-2) T+T-T=(4 n-5) T$. During this time the BS may receive more than $2 n$ frames, but only $2 n$ frames can be counted in the utilization under the fair-access criterion. Minimizing $x$ to achieve the optimal utilization yields $U(2 n) \leq$ $2 n T /(2 n T+(4 n-5) T]=2 n /(6 n-5)$. The rest of the proof is omitted for brevity.

From the proofs of Theorems 1,2, and 3, we can see that we only take use of the knowledge of the topology of sensor nodes within three hops of the base station and the number of frames transferred by them to derive the upper bound of network utilization. Thus, we can extend this analysis method to complex topology network. In Section 8, We will explain it in detail.

\subsection{Bound Achievability via Optimal Fair Scheduling}

In this section, we prove that the performance bounds introduced in Theorems 1, 2, and 3 are indeed achievable. Particularly, we present a TDMA scheduling algorithm that conforms to the fair-access criterion and show that it achieves the performance bounds. Note that herein the optimal utilization is under the constraint of the fair-access criterion. Otherwise, by simply allowing only $O_{n}$ to transmit, the optimal utilization is 1 . Recall that we assume a fixed data frame size and negligible propagation and processing delays. Thus, for the following discussion we divide the time into equal-duration time slots with durations equal to the time needed to transmit one frame. The TDMA algorithm, which we term optimal fair scheduling, is described below.

Optimal fair scheduling for linear topology. Three tables containing the optimal schedules for the cases of $n=1,2,3$, respectively, are shown in Fig. 3 . Each row of the tables depicts node actions in a specific time slot. Consider the examples shown in the table of Fig. $3 \mathrm{~b}$ : at slot $1, O_{1}$ transmits while $\mathrm{O}_{2}$ receives and the BS is idle; at slot 2, $\mathrm{O}_{2}$ relays the frame received in the previous slot to the BS; etc. It is not difficult to show that these schedules achieve the bounds for the cases of $n=1,2,3$, respectively.

For the general case of $n>3$, let $d=D_{\text {opt }}=3(n-1)$. A schedule with cycle $d$ can be created as follows: $O_{1}$ transmits in time slots $(d \cdot j)+1 ; j=0,1, \ldots ; O_{i}(i=2, \ldots, n)$ transmits relayed frames to $O_{i+1}$ from time slot $(d \cdot j)+f(i)$ to 


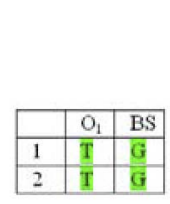

(a) $\mathrm{n}=1$

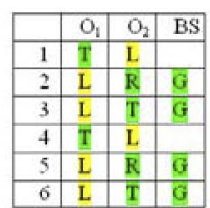

(b) $\mathrm{n}=2$

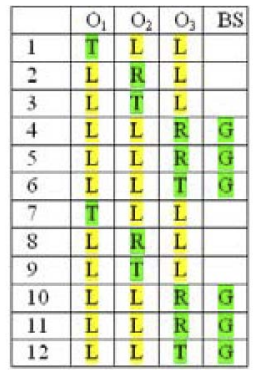

(c) $\mathrm{n}=3$
Fig. 3. Optimal schedules for small linear topologies (Legend: R: relay traffic; T: transmit own traffic; L: listening or receiving: G: frame received at BS).

time slot $(d \cdot j)+f(i)+i-2$ and transmits one of its own frames to $O_{i+1}$ time slot $(d \cdot j)+f(i)+i-1 ; j=0,1, \ldots$, where $f(i)$ is recursively defined as follows:

$$
f(i)= \begin{cases}1, & i=1, \\ f(i-1)+(i-1), & i>1 .\end{cases}
$$

The proof of the schedule's optimality for arbitrary $n$ is omitted for brevity.

Note that if we allow sensors to be self-clocking among sensors by listening to the wireless media, the above TDMA scheme can be implemented easily without requiring system-wide clock synchronization.

Optimal fair scheduling for Fig. 2a grid topology. Before considering a general case, we must first consider some simple cases in which $n$ is small. A schedule for $n=1$ is illustrated in Fig. 4a. The utilization is 1 . With $n=2$, when $O_{11}$ transmits, $O_{12}$ and $O_{22}$ cannot transmit. A schedule is illustrated in Fig. $4 \mathrm{~b}$. The utilization is $4 / 5$. These are consistent with Theorem 2 and are thus optimal.

Optimal fair scheduling for Fig. 2b. We first consider some simple cases where $n$ is small. For Fig. 2b, in which $n=1$, one scheme is shown in Fig. 5a. The utilization is $2 / 3$. For $n=2, O_{12}$ and $O_{11}$ cannot transmit when $O_{21}$ transmits. One possible scheme is shown in Fig. 5b. The utilization is $4 / 7$. With $n=3$, the only nodes that can transmit at the same time are $O_{21}, O_{22}$, and $O_{23}$. One scheme is shown in Fig. $5 c$, and the utilization is $6 / 13$. Each of these is consistent with Theorem 3.

Now consider the general case. To fully utilize parallel transmissions, we let $O_{2 j}(j=0, \ldots, n)$ transmit in the first slot. The second row waits for the remainder of the cycle while the first row forwards the traffic to the BS. This portion is simply a linear topology with double loads.

\begin{tabular}{|c|c|c|c|}
\hline & $\mathrm{O}_{11}$ & $\mathrm{O}_{21}$ & $\mathrm{BS}$ \\
\hline 1 & $\mathrm{~T}$ & $\mathrm{~L}$ & $\mathrm{G}$ \\
\hline 2 & $\mathrm{~L}$ & $\mathrm{~T}$ & $\mathrm{G}$ \\
\hline 3 & $\mathrm{~T}$ & $\mathrm{~L}$ & $\mathrm{G}$ \\
\hline 4 & $\mathrm{~L}$ & $\mathrm{~T}$ & $\mathrm{G}$ \\
\hline
\end{tabular}

(a) $n=1$

\begin{tabular}{|c|c|c|c|c|c|}
\hline & $\mathrm{O}_{11}$ & $\mathrm{O}_{12}$ & $\mathrm{O}_{21}$ & $\mathrm{O}_{22}$ & $\mathrm{BS}$ \\
\hline 1 & $\mathrm{~T}$ & $\mathrm{~L}$ & $\mathrm{~T}$ & $\mathrm{~L}$ & \\
\hline 2 & $\mathrm{~L}$ & $\mathrm{R}$ & $\mathrm{L}$ & $\mathrm{L}$ & $\mathrm{G}$ \\
\hline 3 & $\mathrm{~L}$ & $\mathrm{~T}$ & $\mathrm{~L}$ & $\mathrm{~L}$ & $\mathrm{G}$ \\
\hline 4 & $\mathrm{~L}$ & $\mathrm{~L}$ & $\mathrm{~L}$ & $\mathrm{R}$ & $\mathrm{G}$ \\
\hline 5 & $\mathrm{~L}$ & $\mathrm{~L}$ & $\mathrm{~L}$ & $\mathrm{~T}$ & $\mathrm{G}$ \\
\hline
\end{tabular}

(b) $n=2$
Fig. 4. Optimal schedules for small Fig. 2a grid networks.

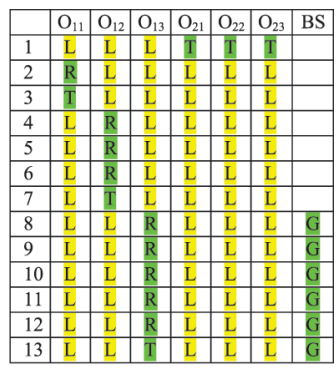

(c) $n=3$ (a) $\mathrm{n}=1$

(b) $n=2$

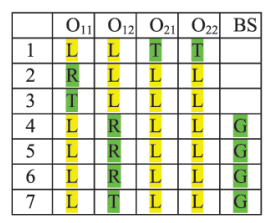

Fig. 5. Optimal schedules for small Fig. 2b grid networks.

Therefore, the achievable utilization is

$$
\frac{2 n}{2 n+2(n-1)+2(n-2)+1}=\frac{2 n}{6 n-5},
$$

which is consistent with Theorem 3. Since the bound is achievable, it is optimal. We can verify Fig. 5 when $n=1,2$, or 3 . Interestingly, when $n \rightarrow \infty$, the asymptotic limit for the upper bound of the optimal utilization is $1 / 3$, which is less than $2 / 3$, or the bound for traffic forwarded across the rows first, as in Fig. 2a.

The optimal scheduling algorithms introduced above, though TDMA in nature, can be implemented without global clock synchronization. This is because a node's reception of a frame originated by its immediate upstream neighbor triggers that node's own transmission for the same cycle, thereby achieving self-clocking.

\subsection{Traffic Load and Sensor Data Sampling Limit}

This section addresses the impact of end-to-end performance bounds on the traffic load limitation of each sensor. Let $\rho$ denote the traffic load generated by each sensor node. For the networks in Figs. 1, 2a, and 2b, since each node can transmit at most one original frame, which requires a period of $T$ in every $3(n-1) T$ time period, $(3 n-1) T$ time period, and $(6 n-5) T$ time period, respectively, we must have $\rho \leq T / x=1 /[3(n-1)], \quad \rho \leq T / x=1 /[(3 n-1)]$, and $\rho \leq$ $T / x=1 /[(6 n-5)]$, respectively, if $n>2$. Furthermore, a data frame contains protocol overhead (because of headers and/or trailers). Thus, $\rho$ must be adjusted to account for this overhead. Denote $\alpha$ to be the fraction of actual data bits in a frame. We have the following three theorems:

Theorem 4. For the linear topology illustrated in Fig. 1, under the fair-access criterion, the maximum feasible per node traffic load is

$$
\frac{\alpha}{3(n-1)}, \text { if } n>2 \text {. }
$$

Theorem 5. For the 2-row grid topology depicted in Fig. 2a, under the fair-access criterion, the maximum feasible per node traffic load is

$$
\frac{\alpha}{3 n-1} \text {, if } n>2 \text {. }
$$

Theorem 6. For the 2-row grid topology depicted in Fig. 2b, under the fair-access criterion, the maximum feasible per node 


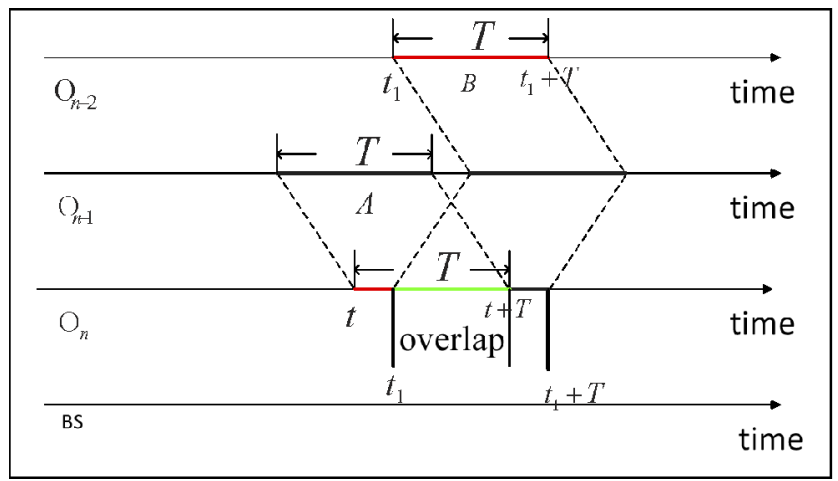

Fig. 6. Overlapping period.

traffic load is

$$
\frac{\alpha}{(6 n-5)}, \text { if } n>2
$$

These three theorems not only tell us the traffic limitation of the sensor network, but they also provide lower bounds on the average sensor sampling rate/intervals (i.e., the minimum supportable time $T / \rho$ between samples). The proofs are omitted.

\section{Underwater Acoustic Sensor Networks}

Consider an underwater sensor network in which the transmission medium is water and the carrier is an acoustic signal. We derive upper bounds on $U(n)$ and lower bounds on the minimum transmission delay, or time between samples, for the linear topology under the fair-access criterion. We consider the impact of nonnegligible propagation delay. We denote transmission time and propagation delay as $T$ and $\tau$, respectively. As stated in the previous section, we let $x$ denote the time period during which the BS successfully receives at least one original data frame from each sensor node in the network. We let $b$ and $y$ denote busy time and idle time, respectively. Thus, we have $x=b+y$. In Theorem 7, we study optimal utilization for underwater sensor networks.

Theorem 7. For the linear topology, under fair access, utilization is upper bounded by the optimal utilization $U_{\text {opt }}(n)$ for all $\tau(\tau \leq T / 2)$

$U(n) \leq U_{\text {opt }}(n)= \begin{cases}n T /[3(n-1) T-2(n-2) \tau], & n>1 \\ 1, & n=1\end{cases}$

and the maximum utilization $U_{\text {opt }}(n)$ can be achieved by a special case. An asymptotic lower limit for the optimal utilization exists and is $1(3-2 \tau / T)$. The intersample time for each node, denoted by $D(n)$, is lower bounded by the minimum effective intertransmission delay for a node, or the minimum cycle time, $D_{\text {opt }}(n)$

$$
D(n) \geq D_{\text {opt }}(n)= \begin{cases}3(n-1) T-2(n-2) \tau, & n>1, \\ T, & n=1 .\end{cases}
$$

Proof of Theorem 7. For $n>2$ : During the time period $x$, the $\mathrm{BS}$ needs to receive at least $n$ frames from $\mathrm{O}_{n}$. Thus, $\mathrm{O}_{n}$ transmits at least $n$ frames (including $n-1$ relayed frames and one of its generated frames). We have

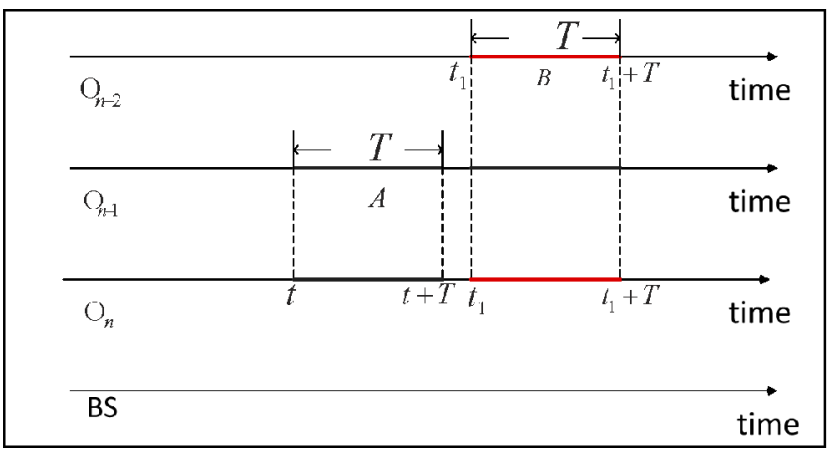

Fig. 7. Idle period in terrestrial wireless sensor network.

$b \geq n T$. Likewise, in order for $\mathrm{O}_{n}$ to receive $(n-1)$ frames from $\mathrm{O}_{n-1}, \mathrm{O}_{n}$ needs to listen to at least $(n-1)$ frames, during this time (there is $\tau$ time delay) the BS must be idle. In the proof for terrestrial wireless sensor networks, since the propagation delay is ignored, when $\mathrm{O}_{n-2}$ transmits, $\mathrm{O}_{n}$ cannot receive frames from $\mathrm{O}_{n-1}$ because $\mathrm{O}_{n-1}$ cannot transmit and receive frames at the same time. However, in underwater sensor network in which propagation delay cannot be ignored, when $\mathrm{O}_{n-2}$ transmits, $\mathrm{O}_{n}$ still can receive frames from $\mathrm{O}_{n-1}$. This fact is illustrated be the example in Fig. 6. As shown in Fig. 6, we assume that $\mathrm{O}_{n}$ receives frame $A$ in $(t, t+T)$ and $\mathrm{O}_{n-2}$ transmits frame $B$ in $\left(t_{1}, t_{1}+T\right)$. Since $\mathrm{O}_{n-2}$ and $\mathrm{O}_{n}$ are within two-hops, $\mathrm{O}_{n}$ is blocked in $\left(t_{1}, t_{1}+T\right)$ assuming the propagation delay is the same between both node pairs. For example, the overlap is $\left(t_{1}, t+T\right)$ in Fig. 6. In other words, when $\mathrm{O}_{n-2}$ transmits in $\left(t_{1}, t+T\right)$, $\mathrm{O}_{n}$ can still receive frames. As illustrated in Fig. 7, in terrestrial wireless sensor networks, $\mathrm{O}_{n}$ cannot transmit when either $\mathrm{O}_{n-1}$ or $\mathrm{O}_{n-2}$ is transmitting. Furthermore, when $\mathrm{O}_{n-2}$ transmits $B, \mathrm{O}_{n-1}$ cannot transmit $A$. Thus, the idle period generated by $\mathrm{O}_{n-2}$ transmitting $B$ and $\mathrm{O}_{n-1}$ transmitting $A$ is $2 T$. However, as shown in Fig. 6, in underwater sensor networks, the idle period generated by $\mathrm{O}_{n-2}$ transmitting $B$ and $\mathrm{O}_{n-1}$ transmitting $A$ is $t_{1}+T-t$, which is less than $2 T$.

Under the constraint of $\tau \leq T / 2$, when overlapping is maximized, the idle period generated independently by frame $B$ reaches its minimum. To maximize the throughput of $\mathrm{O}_{n-1}$, let $\mathrm{O}_{n-1}$ first finish transmitting frame $A$, then begin receiving of frame $B$ immediately.

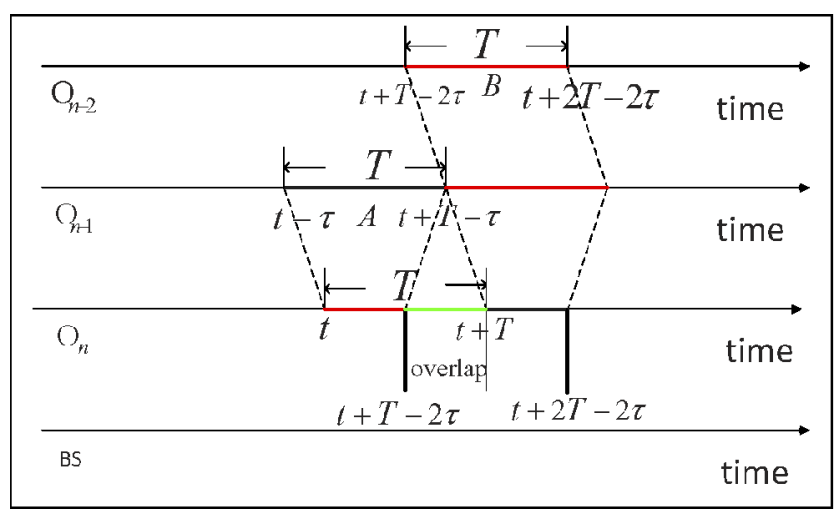

Fig. 8. Maximal overlapping $(\tau \leq T / 2)$. 


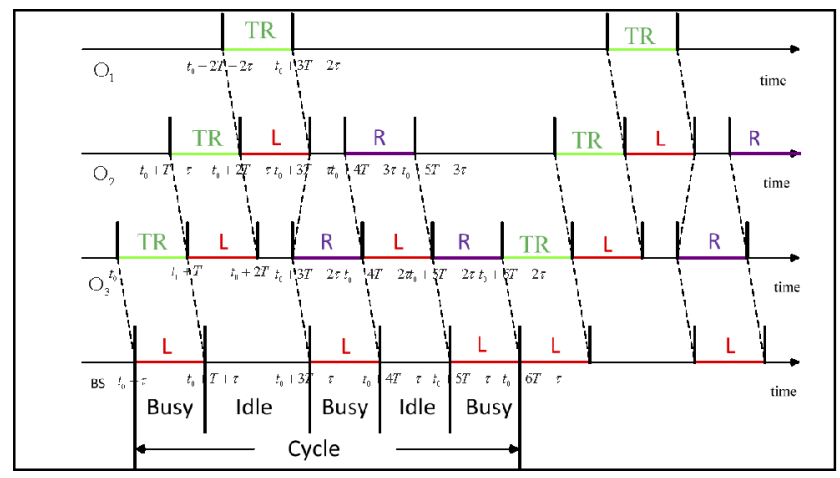

Fig. 9. Bottom-up approach for Linear topology $(n=3)$ [Legend: TR: transmit own traffic; R: relay traffic (note: actually relay latest received frame from upstream nodes); L: receiving].

This analysis is illustrated in Fig. 8: $\mathrm{O}_{n}$ receives frame $A$ in $(t, t+T)$, which implies that $\mathrm{O}_{n-1}$ transmitted frame $A$ in $(t-\tau, t-\tau+T)$. Let $\mathrm{O}_{n-2}$ transmit frame $B$ in $(t+$ $T-2 \tau, t+2 T-2 \tau)$ so that its first bit reaches $\mathrm{O}_{n-1}$ in $t+T-\tau$. From Fig. 8, it is easy to see that, if $T-2 \tau \geq 0$, for $\tau \leq T / 2$, the maximum overlapping period is $(t+T-2 \tau, t+T)$. Thus, the minimum time during which $\mathrm{O}_{n}$ may not transmit in order to prevent collision with frame $B$ at $\mathrm{O}_{n-1}$ is $(t+2 T-2 \tau)-(t+T)=T-2 \tau$. Therefore, the total time in which $\mathrm{O}_{n}$ must be idle, assuming that each frame is sent individually, is $y v \geq$ $(n-1) T+(n-2)(T-2 \tau)$. Therefore, we have $x=b+$ $y \geq n T+(n-1) T+(n-2)(T-2 \tau)=(n-1)(3 T-2 \tau)+2 \tau$.

Since $D(n)=x$, we are able to derive (13) for the case of $n>2$. During the time period $x$, the BS may receive more than $n$ frames, but only $n$ frames can be counted in the utilization under the fair-access criterion. Since we must minimize $x$ to achieve the optimal utilization, we have

$$
\begin{aligned}
U(n) & \leq n T /[n T+(n-1) T+(n-2)(T-2 \tau)] \\
& =n T /[(n-1)(3 T-2 \tau)+2 \tau],
\end{aligned}
$$

which proves (13) for the case of $n>2$.

For $n=2$ : Since we want $G_{1}=G_{2}$ during the time period $x, \mathrm{O}_{2}$ transmits at least two frames (one relayed frame and its own). We have $b \geq 2 T . \mathrm{O}_{2}$ needs to listen to at least one frame from $\mathrm{O}_{1}$. We have $y \geq T$ and thus $x=b+y \geq 3 T$. Therefore, we must minimize $x$ to achieve the optimal utilization, $U(n)=2 T / x \leq 2 T / 3 T=2 / 3$, which proves (13) for this case. Note that the propagation delay can be ignored since it is possible to send the frame from $\mathrm{O}_{1}$ such that it arrives at $\mathrm{O}_{2}$ just as $\mathrm{O}_{2}$ finishes transmitting of the previous frames.

For $n=1$ : Obviously, $U(1) \leq 1$. We will prove that the performance bounds $U_{\text {opt }}(n)$ are indeed achievable in a special case in the next section.

Note that herein the optimal utilization is under the constraint of the fair-access criterion when $\tau \leq T / 2$. We first give the algorithm for the optimal fair scheduling. We then show the optimal fair scheduling for the cases of $n=3,5$ in Figs. 9 and 10, respectively. Before showing the algorithm, we must provide some notation. Let $A_{i}$ denote the frame generated by $\mathrm{O}_{i}$, where $1 \leq i \leq n$.

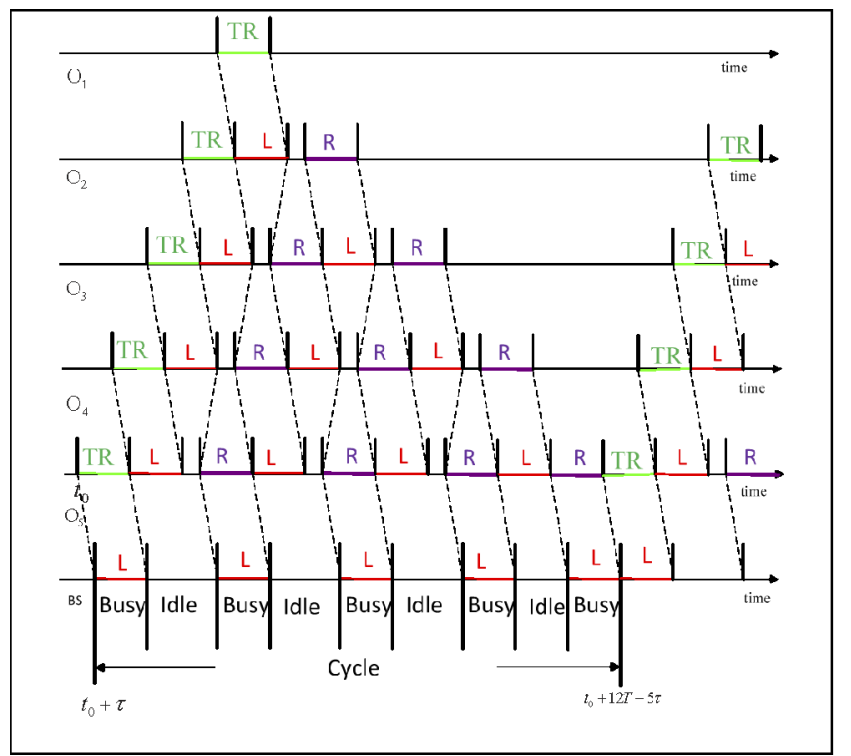

Fig. 10. Bottom-up approach for Linear topology $(n=5)$ [Legend: the same].

Algorithm for optimal fair scheduling for linear topology. First, we define a cycle. Let $t_{0}$ denote the time when $\mathrm{O}_{n}$ begins transmission of its own frame, $A_{n}$. Thus, the BS receives frame $A_{n}$ from time $t_{0}+\tau$. As we mentioned above, $x$ is the cycle time for the network under the fair-access criterion. Thus, we define a cycle as $\left(t_{0}+\tau, t_{0}+\tau+x\right)$. Therefore, the next cycle is $\left(t_{0}+\tau+x, t_{0}+\tau+2 x\right)$.

Second, for any node $\mathrm{O}_{i}$, in which $1 \leq i \leq n$ in the cycle $\left(t_{0}+\tau, t_{0}+\tau+x\right)$, it has a start time (the time at which starts to transmit its own frame, $A_{i}$ ) and an end time (the time at which $\mathrm{O}_{i}$ just completes $A_{1}$ 's transmission). We denote the start and end times by $s_{i}$ and $d_{i}$, respectively. $s_{i}$ and $d_{i}$ are defined as follows:

$$
\begin{gathered}
s_{i}= \begin{cases}t_{0}+(n-i) T-(n-i) \tau & 1 \leq i<n \\
t_{0} & i=n,\end{cases} \\
d_{i}= \begin{cases}s_{i}+T+(i-1)(3 T-2 \tau) & 1 \leq i<n \\
t_{0}+x & i=n,\end{cases}
\end{gathered}
$$

where $x=3(n-1) T-2(n-2) \tau$.

Third, we define $\left(s_{i}, d_{i}\right)$ as an active period for node $\mathrm{O}_{i}$, in which $(1 \leq i \leq n)$ is in the cycle $\left(t_{0}+\tau, t_{0}+\tau+x\right)$. In the period $\left(s_{i}, d_{i}\right), \mathrm{O}_{i}$ includes a TR (transmit own traffic) period and $i-1$ subcycles. Their definitions are given as follows: $\left[s_{i}, s_{i}+T\right]$ denotes the TR period during which $\mathrm{O}_{i}$ transmits its own frame $A_{i} ;\left[s_{i}+T, d_{i}\right]$ is divided into $i-1$ subcycles; we denote a subcycle by $\left[u_{i, j}, u_{i, j+1}\right], j=1, \ldots, i-1$, during which time $\mathrm{O}_{i}$ receives and relays a frame from each upstream node. Thus, we have

$$
\left\{\begin{array}{l}
u_{i, 1}=s_{i}+T \\
u_{i, j}=(j-1)(3 T-2 \tau)+u_{i, 1} \quad j=2, \ldots, i-1 \\
u_{i, i}=d_{i} .
\end{array}\right.
$$

Finally, for any subcycle $\left[u_{i, j}, u_{i, j+1}\right]$, there are three phases. We give them as follows: In phase $\left[u_{i, j}, u_{i, j}+T\right], \mathrm{O}_{i}$ receives a frame from $\mathrm{O}_{i-1}$, where $2 \leq i \leq n$; in phase $\left[u_{i, j}+T, M\right], \mathrm{O}_{i}$ is idle (neither receiving a frame nor transmitting a frame), where 


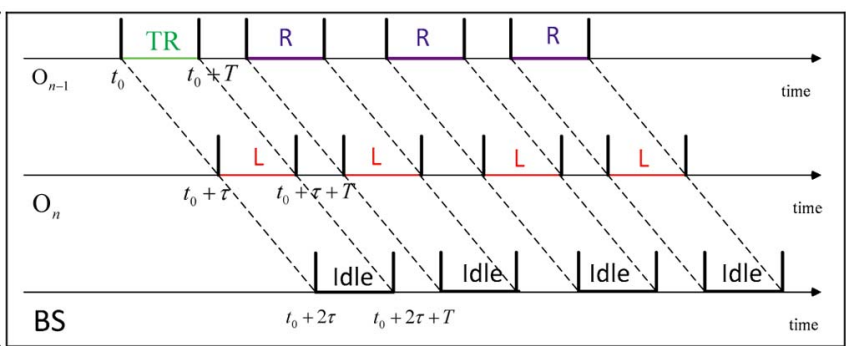

Fig. 11. Idle period generated by $\mathrm{O}_{n-1}$ 's transmission.

$$
M= \begin{cases}u_{i, j}+T & i=n \text { and } j=n-1 \\ u_{i, j}+T+T-2 \tau & \text { others; }\end{cases}
$$

in phase $\left[M, u_{i, j+1}\right]$, where $u_{i, j+1}=M+T, \mathrm{O}_{i}$ relays a frame to $\mathrm{O}_{i+1}$, where $2 \leq i \leq n$. Note, when $i=n, \mathrm{O}_{n+1}$ represents the base station.

Two examples of this schedule are illustrated in Figs. 9 and 10. We show the case in which $n=3$ in Fig. 9. The cycle period is $6 T-2 \tau$, and the utilization of the BS is $3 T / 6 T-2 \tau$, which is consistent with Theorem 7 . The theorem also holds for the case in which $n=5$, as shown in Fig. 10, where the cycle period is $12 T-6 \tau$ and the utilization of the BS is $5 T / 12 T-6 \tau$. For the case of $\mathrm{n}$ nodes, it is easy to verify this (omitted). Thus, the performance bounds are indeed achievable in a special case under the algorithm above.

Theorem 8. For the linear topology, under fair access, $U(n)$ is upper bounded by $n T /[n T+(n-1) T]$ for all $\tau(\tau>T / 2)$.

Proof of Theorem 8. For $n>2$ : During the time period $x$, the BS needs to receive at least $\mathrm{n}$ frames from $\mathrm{O}_{n}$ (including $n-1$ relayed frames and one of its generated frames). Thus, $\mathrm{O}_{n}$ transmits at least $n$ frames. We have $b \geq n T$. In order for $\mathrm{O}_{n}$ to receive $(n-1)$ frames from $\mathrm{O}_{n-1}, \mathrm{O}_{n}$ needs to listen for at least $(n-1)$ frames during which time $\mathrm{O}_{n}$ cannot transmit. Thus, there exists $(n-1) T$ corresponding idle periods in the base station. This fact is illustrated in Fig. 11. $\mathrm{O}_{n-1}$ transmits a frame in $\left(t_{0}, t_{0}+T\right)$, then $\mathrm{O}_{n}$ receives it in $\left(t_{0}+\tau, t_{0}+\right.$ $\tau+T)$ since there is propagation delay $\tau$. Thus, no frame will arrive base station in $t_{0}+2 \tau, t_{0}+2 \tau+T$. Therefore, during the time period $x$, we have $y \geq(n-1) T$. Therefore, we have the following inequality: $x=b+y \geq n T+(n-1) T=(2 n-1) T$. Since we must minimize $x$ to achieve the optimal utilization, we have $U(n) \leq n T /[n T+(n-1) T]=n /(2 n-1)$.

For $n=2$ : Since we want $G_{1}=G_{2}$ during the time period $x, \mathrm{O}_{2}$ transmits at least two frames (one relayed frame and its own). We have $b \geq 2 T$. $\mathrm{O}_{2}$ needs to listen to at least one frame from $\mathrm{O}_{1}$. We have $y \geq T$, and thus $x=b+y \geq 3 T$. So, minimizing $x$ yields the optimal utilization, $U(n)=2 T / x \leq 2 T / 3 T=2 / 3$, which proves the inequality for this case.

For $n=1$. Obviously, $U(1) \leq 1$.

Next, we address the impact of end-to-end performance bounds on the traffic load limitation of each sensor. Let $\rho$ denote the traffic load generated by each sensor node. We express the propagation delay, $\tau$, in normalized time units as $\alpha=\tau / T$. For a linear network under the constraint of the criterion, since each node can transmit at most one original frame, which requires a period of $T$ in every $3(n-1) T-2(n-2) \tau$ time period, we must have $\rho \leq T / x=$ $1 /[3(n-1)-2(n-2) \alpha]$, where $0 \leq \alpha \leq 1 / 2$ if $n \geq 2$. Denote $m$ as the fraction of actual data bits in a frame. We have the following theorem:

Theorem 9. For the linear topology, under the fair-access criterion, for all $\tau(\tau \leq T / 2)$, the maximum feasible per node traffic load is $m /[3(n-1)-2(n-2) \alpha]$ if $n \geq 2$.

Next, we consider the energy consumption aspect $E(n)(\tau \leq T / 2)$. Let $B_{T}, B_{R}, B_{L}$, and $B_{S}$ denote the energy consumption per unit of time for a node to transmit a frame or to receive a frame, when a node is listening, and when a node is sleeping, respectively. It is reasonable to assume that $B_{T}>B_{R} \geq B_{L}>B_{S}$. Let $E(n), E_{T}(n), E_{R}(n), E_{L}(n)$, and $E_{S}(n)$ denote the energy consumption, the transmission energy consumption, the reception energy consumption, the listening energy consumption, and the sleeping energy consumption, respectively, for the linear topology under fair access in a cycle. Let $E_{i}(n)$ denote node $\mathrm{O}_{i}{ }^{\prime} \mathrm{s}$ energy consumption in a cycle.

Theorem 10. For the linear topology, under fair access, $E(n)$ is lower bounded by the minimum energy consumption, $E_{\text {opt }}(n)$ when $\tau \leq T / 2$ :

$$
\begin{aligned}
E_{\text {opt }}(n)= & \sum_{i=1}^{n}\left(B_{T} i T+B_{R}(i-1) T\right. \\
& \left.\left.+B_{L}((3 n-2 i-2) T-2(n-2) \tau)\right)\right) .
\end{aligned}
$$

Moreover, according to the Algorithm for Optimal Fair Scheduling for Linear Topology in Theorem 7, we can let nodes sleep when they neither transmit nor receive frames. Therefore, the more efficient energy consumption $\hat{E}_{\text {opt }}(n)$ :

$$
\begin{aligned}
\hat{E}_{\text {opt }}(n)= & \sum_{i=1}^{n}\left(B_{T} i T+B_{R}(i-1) T\right. \\
& \left.\left.+B_{S}((3 n-2 i-2) T-2(n-2) \tau)\right)\right) .
\end{aligned}
$$

Proof of Theorem 10. Let $E(n)$ denote the total energy consumption for the linear topology which includes $n$ nodes. It is easy to see that $E(n)=\sum_{i=1}^{n} E_{i}(n)$. Since $E_{\text {opt }}(n)=\min (E(n)), E_{\text {opt }}(n)=\min \left(\sum_{i=1}^{n} E_{i}(n)\right)$. Since $E_{i}(n) \geq 0$ for $i=1,2, \ldots n$, we have $E_{\text {opt }}(n)=\sum_{i=1}^{n} \min \left(E_{i}(n)\right)$ Therefore, we only need to determine the $\min \left(E_{i}(n)\right)$. For any node $\mathrm{O}_{i}(1 \leq i \leq n)$ in a cycle, we have $E_{i}(n)=$ $B_{T} T_{T}+B_{R} T_{R}+B_{L} T_{L}$, where $T_{T}$ denotes the period during which $\mathrm{O}_{i}$ transmits frames in a cycle, $T_{R}$ denotes the period during which $\mathrm{O}_{i}$ receives frames in a cycle, and $T_{L}$ denotes the period during which $\mathrm{O}_{i}$ listens in a cycle. Thus, it is easy to see that $\min \left(E_{i}(n)\right)=$ $\min \left(B_{T} T_{T}+B_{R} T_{R}+B_{L} T_{L}\right)=\min \left(B_{T} T_{T}\right)+\min \left(B_{R} T_{R}\right)+$ $\min \left(B_{L} T_{L}\right)$, where $x=T_{T}+T_{R}+T_{L}$ and $B_{T}>B_{R} \geq B_{L}$. First, we consider the $B_{T} T_{T}$, as we know $B_{T}$ is a positive constant parameter. Therefore, we only need to get the minimum of $T_{T}$. Since $\mathrm{O}_{i}$ transmits at least $i$ frames (including $i-1$ relayed frames and one of its generated frames) during a cycle, we have $T_{T} \geq i T$. Thus, $\min \left(B_{T} T_{T}\right)=B_{T} i T$. As mentioned above, $\mathrm{O}_{i}$ relayed at least $i-1$ frames, meaning that $\mathrm{O}_{i}$ receives at least 
(a) Optimal utilization

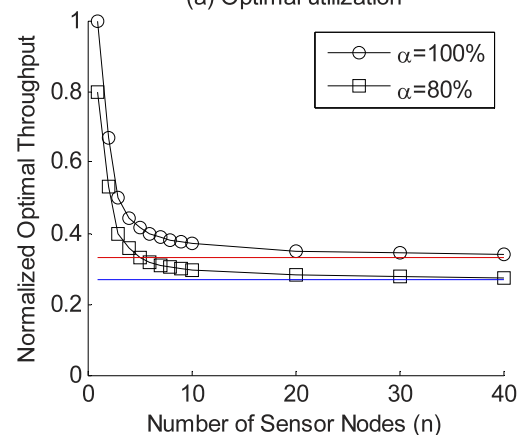

(b) Average Dealy

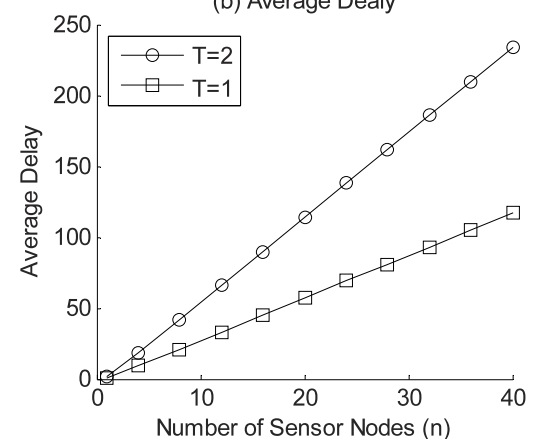

(c) Per node load limit

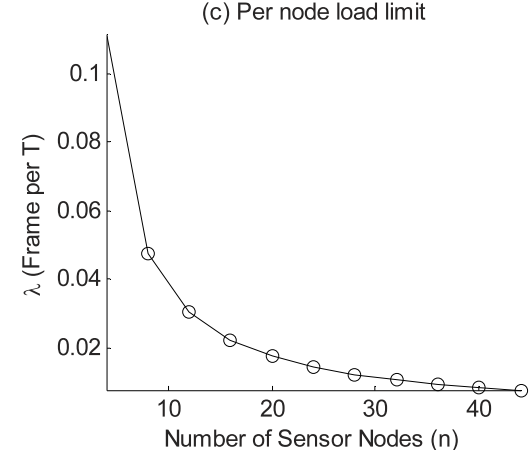

Fig. 12. Performance in linear topology (a) Optimal utilization, (b) Delay, and (c) Per node load limit.

$i-1$ frames. We have $T_{R} \geq(i-1) T$. Thus, we have $\min \left(B_{R} T_{R}\right)=B_{R}(i-1) T$. Likewise, $B_{L}$ is also a positive constant parameter, therefore, we only need to know the minimum of $T_{L}$ under the constraint $x=T_{T}+T_{R}+T_{L}$. Therefore, we have $T_{L}=x-T_{T}+T_{R}$. From Theorem 7, we have $x=b+y \geq n T+(n-1) T+(n-2)(T-2 \tau)$. Thus, we have $T_{L} \geq(3 n-2 i-2) T-2(n-2) \tau$. Therefore, we have $\min \left(E_{i}(n)\right)=B_{T} i T+B_{R}(i-1) T+B_{L}[(3 n-2 i-2) T-$ $2(n-2) \tau]$. Therefore, we have $E_{\text {opt }}(n)=\sum_{i=1}^{n} \min \left(E_{i}(n)\right)=$ $\left.\sum_{i=1}^{n}\left(B_{T} i T+B_{R}(i-1) T+B_{L}((3 n-2 i-2) T-2(n-2) \tau)\right)\right)$. We want to reduce energy consumption further. According to the Algorithm for Optimal Fair Scheduling for Linear Topology in Theorem 3, nodes sleep during the period in which they should listen. Thus, we have $\hat{E}_{\text {opt }}(n)=\sum_{i=1}^{n} \min \left(E_{i}(n)\right)=\sum_{i=1}^{n}\left(B_{T} i T+B_{R}(i-1) T+\right.$ $\left.\left.B_{S}((3 n-2 i-2) T-2(n-2) \tau)\right)\right)$.

\section{Performance Evaluation of RF-Based WSNs}

In this section, we provide some projected performances for WSNs (nonunderwater). To account for protocol overhead, the optimal utilizations have been multiplied by $\alpha$, which is the fraction of actual data bits in a data frame.

\subsection{Linear Topology}

Fig. 12a shows the optimal utilization versus the number of nodes for different $\alpha$ values for the basic linear topology based on the bounds of Theorem 1. The optimal utilization decreases quickly as $n$ increases and approaches the asymptotic lower limit of optimal utilization, as suggested by the theorem. When $n=5$, the optimal utilization is already near the asymptotic bound, which is indicated by the horizontal, colored lines.

Figs. $12 \mathrm{~b}$ and $12 \mathrm{c}$ show the more significant impacts on linear topologies of increasing the network size. The minimum average delay increases linearly with $n$, as shown in Fig. 12b. The traffic limit per sensor node decreases quickly as $n$ increases, as shown in Fig. 12c, and approaches the asymptotic limit of zero.

\subsection{Grid Topology}

Fig. 13a shows the optimal utilization versus $n$ for different $\alpha$ values in the two-row topologies of Fig. 2, as derived from Theorems 2 and 3. Fig. 13a shows that the topology of Fig. 2a may achieve much better utilization than the topology of
Fig. 2b. The delay and load characteristics of the two-row grid topology are illustrated by Figs. 13b and 13c.

\subsection{Linear Topology versus 2-Row Grid}

Fig. 14 compares the optimal utilization of the linear topology of Fig. 1 with that of the horizontal-first-forwarding 2-row grid of Fig. 2a. It is noteworthy that the optimal utilization of the Fig. 2a topology is better than that of the one in Fig. 1, due to parallel transmissions of diagonal neighbors. This suggests that a 2-row grid may be preferable to a linear topology for some applications in which a linear topology might have been the first consideration. This issue is left for further study. Note, however, that the vertical-first grid (Fig. 2b) actually performs worse in terms of network utilization, albeit insignificantly, than the linear topology.

\section{Performance Evaluation of ACOUSTIC-BASED UASNS}

In this section, due to limited space, we present some selected results for underwater sensor networks. To account for protocol overhead, the optimal utilizations have been multiplied by $m$, which is the fraction of actual data bits in a frame. We define the propagation delay factor as $\alpha=\tau / T$.

Fig. 15a shows the optimal utilization versus the propagation delay factor $(\alpha)$ for different $n$ values (number of nodes) when $m=1$ based on the bounds of Theorem 3 . We can see that for $\alpha=0.5$, the throughput achieves maximum in this range of $\alpha$ for different $n$ values. When $n$ goes to infinity, the limit is $1 /(3-2 \alpha)$.

Figs. $15 \mathrm{~b}$ and $15 \mathrm{c}$ show the optimal utilization versus the number of nodes when $m=1$ and $m=0.8$, respectively, for different $\alpha$ values based on the bounds of Theorem 3. The optimal utilization decreases quickly as $n$ increases and approaches the asymptotic lower limit of optimal utilization, as suggested by the theorem. We can also see that for $\alpha=0.5$, the throughput achieves maximum in this range of $\alpha$.

Fig. 16a shows that the effective transmission delay increases linearly with $n$ for different $\alpha$ values. Fig. 16b shows that the traffic limit per sensor node decreases quickly as $n$ increases for different $\alpha$ values, and approaches the asymptotic limit of zero.

Fig. 17a shows the optimal energy consumption versus the propagation delay factor when $n=10, B_{R}=B_{L}$, and $B_{R} / B_{T}=1 / 2$. We observe that different nodes have equal tendencies to decrease energy consumption as the factor 

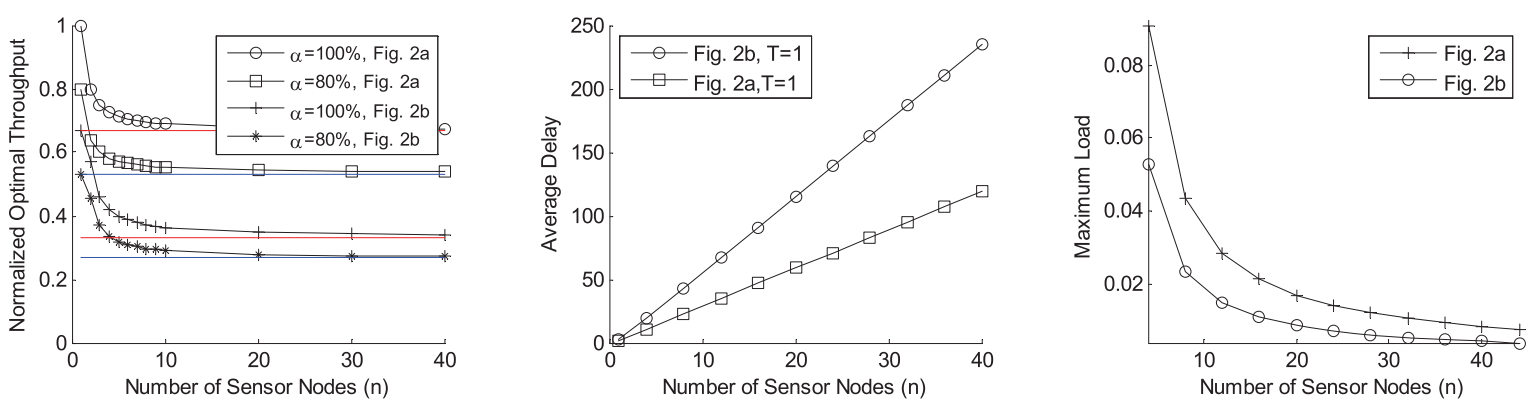

Fig. 13. Performance in 2-row grid (a) Optimal utilization, (b) Min cycle time, and (c) Max per node load.

increases and that the down stream nodes consume more energy.

Fig. 17b shows the optimal energy consumption versus the propagation delay factor when $n=10, B_{R}=B_{L}$, and $B_{R} / B_{T}=2 / 3$. We observe that different nodes have equal tendencies to decrease energy consumption as the factor increases and that the downstream nodes consume more energy.

Fig. 17c shows the optimal energy consumption versus $r=B_{R} / B_{T}$ when $n=10, B_{R}=B_{L}$, and $\alpha=0.25$. Fig. $17 \mathrm{c}$ shows that energy consumption increases as $r=B_{R} / B_{T}$ increases. Also, as the ratio approaches 1 , energy consumption of different nodes will be equal.

Fig. $17 \mathrm{~d}$ shows the optimal energy consumption versus $r=B_{R} / B_{T}$ when $n=10, B_{R}=B_{L}$, and $\alpha=0.5$. Fig. $17 \mathrm{~d}$ shows that energy consumption increases as $r=B_{R} / B_{T}$ increases. Also, as the ratio approaches 1, energy consumption of different nodes will be equal.

\section{Analysis of Bounds in More Complex TOPOLOGIES}

\subsection{RF-Based Wireless Sensor Network (Nonunderwater)}

In this section, we show how to obtain the performance bounds of more complex topologies using the analysis mentioned in Theorems 1,2, and 3. Note that obtained

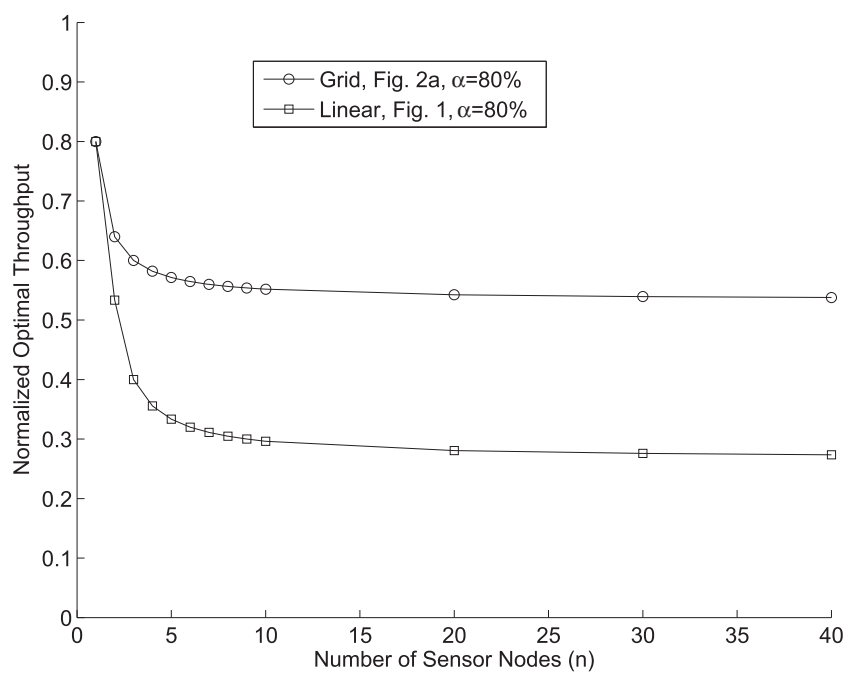

Fig. 14. Optimal utilization (linear versus 2-row grid of Fig. 2a). bounds from this analysis in this section may not be tight. As for tight bounds, we must have knowledge of entire network topologies and routing patterns such that we can design a scheduling algorithm to achieve them. In our analysis method, no node, including the base station, needs to be aware of the entire network topology. The only knowledge we need in this analysis is given as follows:

- The topology of nodes within three hops of the base station;

- Nodes within three hops of the base station must know how many nodes need them to transfer frames to the base station.

According to the above two rules, a complicated topology can be simplified. For example, to obtain the performance bounds of networks like the one in Fig. 18a, the only knowledge that we need to know is illustrated in Fig. 18b. From Fig. 18b, the number of nodes which need node $e$ to transfer their frames is 6 and the number of nodes which need node $f$ to transfer their frame is 5 . In the following, we apply this analysis method to a $k \times n$ grid network. Data frames are forwarded along parallel rows in this grid network, as illustrated in Fig. 19. When $k$ is odd, let $k=2 m+1$, where $m=0,1,2 \ldots$ As illustrated in Fig. 19a, only nodes $\mathrm{O}_{1 n}, \mathrm{O}_{2 n}$, and $\mathrm{O}_{3 n}$ can transfer data frames to BS directly. Likewise, when $k$ is even, let $k=2 m$, where $m=$ $1,2 \ldots$ As illustrated in Fig. 19b, only nodes $\mathrm{O}_{1 n}$ and $\mathrm{O}_{2 n}$ can transfer data frames to the BS directly. We discuss performance upper bounds for this general grid network based on the value of $k$.

Case 1: $\mathbf{k}=1$. When $k=1$, the general grid network was reduced to the linear topology given in Fig. 1. The only knowledge we need to obtain the upper bound on network utilization is given in Fig. 20. From Fig. 20, during the time period $x, \mathrm{O}_{1(n-2)}$ needs to transmit at least $n-2$ frames. The analysis method is given in Theorem 1 . Thus, the upper bound on network utilization for the case $k=1$ is $U(n) \leq n /[3(n-1)]$.

Case 2: $k=2$. When $k=2$, the general grid network was reduced to the 2-row grid topology given in Fig. 2a. The knowledge that we need to obtain the upper bound is given in Fig. 21. During the time period $x$, both $\mathrm{O}_{1(n-2)}$ and $\mathrm{O}_{2(n-2)}$ need to transmit at least $n-2$ frames. Also, the analysis method to get the upper bound is given in Theorem 2. Thus, the upper bound on network utilization for case $k=2$ is $U(2 n) \leq 2 n /(3 n-1)$.

Case 3: $k=3$. For a 3-row grid topology network, the knowledge that we need to obtain the upper bound is given 

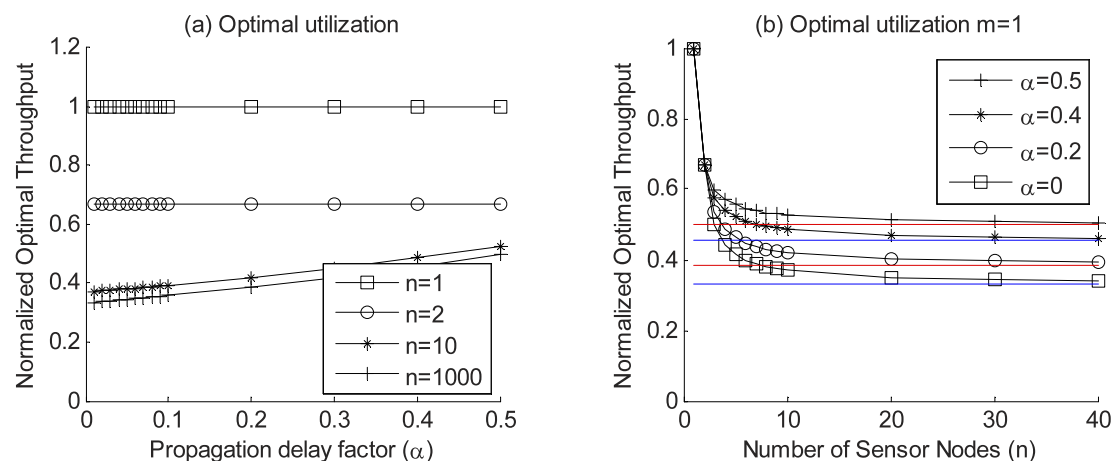

(c) Optimal utilization $m=0.8$

Fig. 15. Optimal utilization.
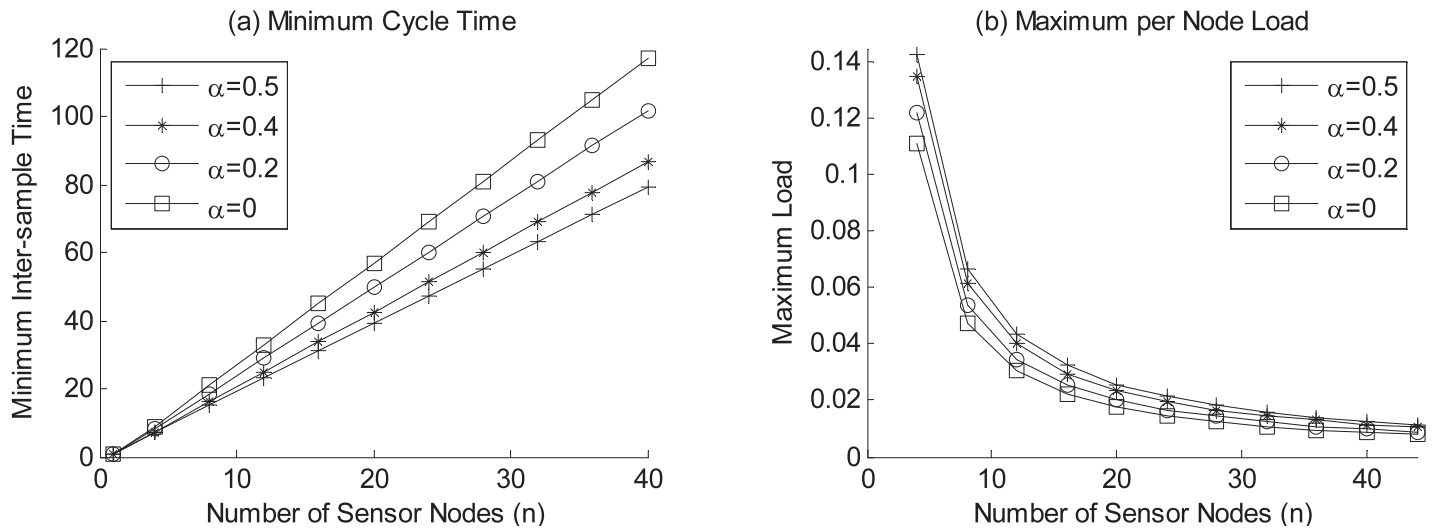

Fig. 16. (a) Minimum cycle time, (b) Maximum per node load.
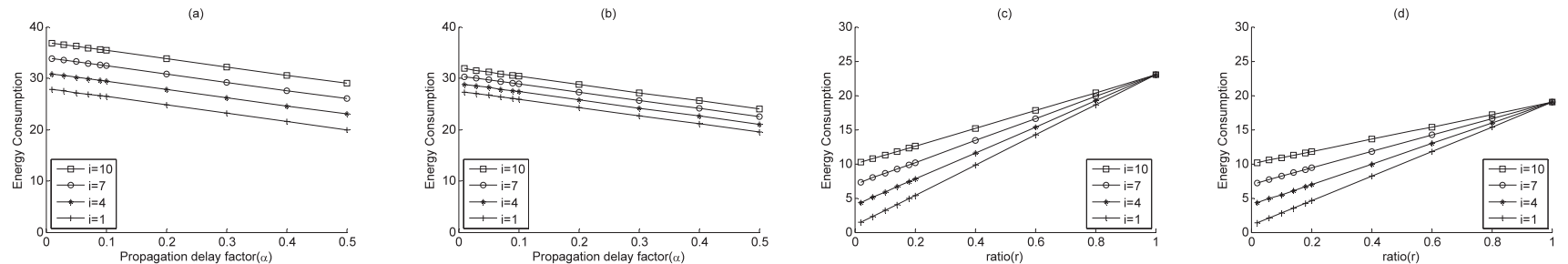

Fig. 17. (a) Optimal energy consumption, (b) Optimal energy consumption, (c) Optimal energy consumption versus r, and (d) Optimal energy consumption versus $r$.

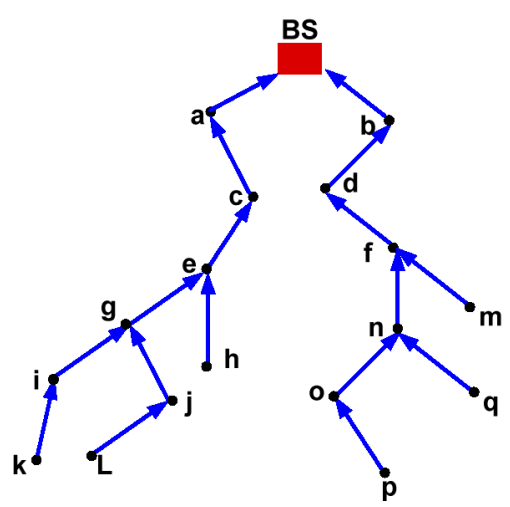

(a) Entire Topology

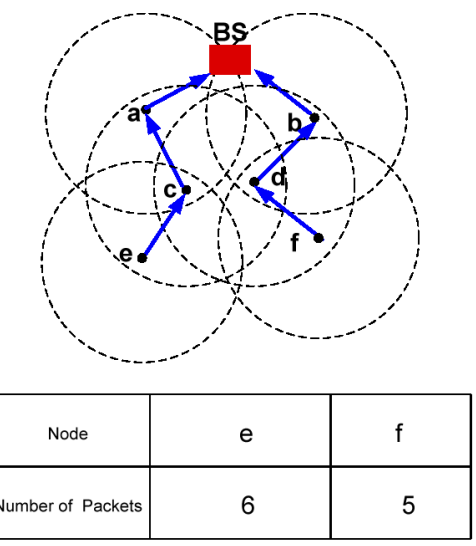

(b) Reduced Topology

Fig. 18. Demonstration of network topology simplification.

in Fig. 22. During the time period $x, \mathrm{O}_{1(n-2)}, \mathrm{O}_{2(n-2)}$, and $\mathrm{O}_{3(n-2)}$ need to transmit at least $n-2$ frames. Note that, when $\mathrm{O}_{1(n-2)}$ transmits, $\mathrm{O}_{1 n}$ cannot transmit but either $\mathrm{O}_{2 n}$ or $\mathrm{O}_{3 n}$ can. Similarly, when $\mathrm{O}_{2(n-2)}$ transmits, $\mathrm{O}_{2 n}$ cannot transmit but either $\mathrm{O}_{1 n}$ or $\mathrm{O}_{3 n}$ can. When $\mathrm{O}_{3(n-2)}$ transmits,
$\mathrm{O}_{3 n}$ cannot transmit but either $\mathrm{O}_{1 n}$ or $\mathrm{O}_{2 n}$ can. Under the fairaccess criterion, $\mathrm{O}_{1 n}, \mathrm{O}_{2 n}$, and $\mathrm{O}_{3 n}$ each need to transmit at least $n$ frames to the BS. We have $b \geq 3 n T$. In order for $\mathrm{O}_{1 n}$ to receive $n-1$ frames from $\mathrm{O}_{1(n-1)}, \mathrm{O}_{1 n}$ needs to listen to at least $n-1$ frames, during which time $\mathrm{O}_{2 n}$ cannot transmit 


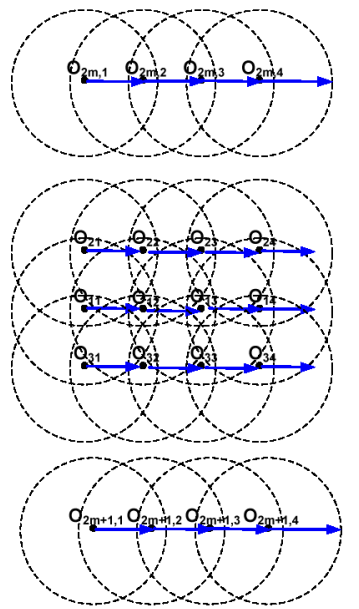

(a) $k$ is odd
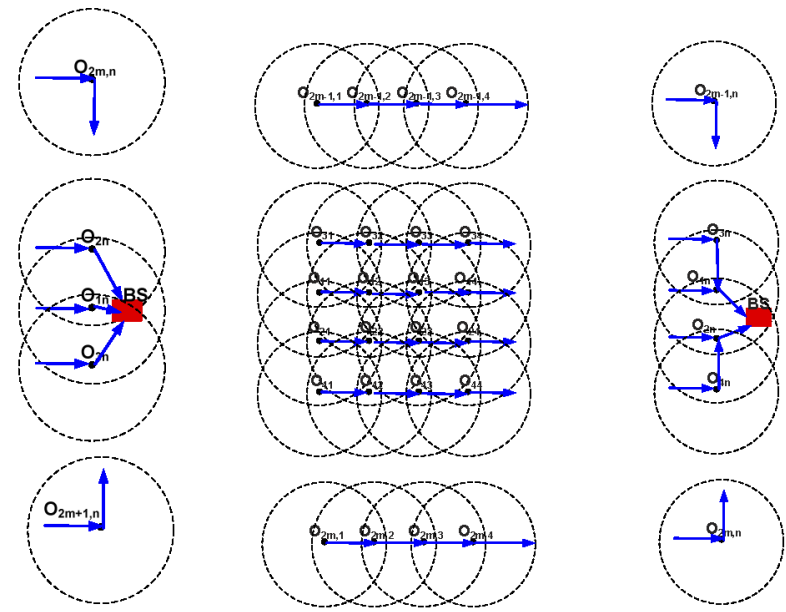

(b) $k$ is even

Fig. 19. General grid network.

but it can receive and $\mathrm{O}_{3 n}$ can either transmit or receive. Similarly, in order for $\mathrm{O}_{3 n}$ to receive $n-1$ frames from $\mathrm{O}_{3(n-1)}, \mathrm{O}_{3 n}$ needs to listen to at least $n-1$ frames during which time $\mathrm{O}_{2 n}$ cannot transmit but it can receive, and $\mathrm{O}_{1 n}$ can either transmit or receive. But in order for $\mathrm{O}_{2 n}$ to receive $n-1$ frames from $\mathrm{O}_{2(n-1)}, \mathrm{O}_{2 n}$ needs to listen to at least $n-1$ frames, during which time neither $\mathrm{O}_{1 n}$ nor $\mathrm{O}_{3 n}$ can transmit, which means that the BS must be idle. Thus, $y \geq(n-1) T$. The upper bound on network utilization is $U(3 n) \leq$ $3 n /(3 n+n-1)=3 n /(4 n-1)$. In our previous work, we proved this upper bound can be achieved by a scheduling algorithm [18]. Thus, this bound is tight.

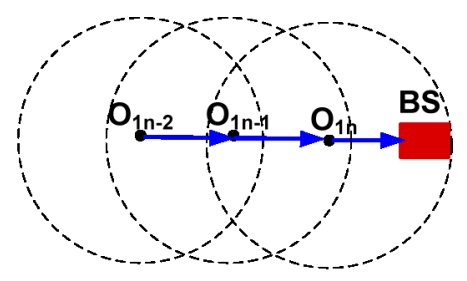

\begin{tabular}{|c|c|}
\hline Node & $\mathrm{O}_{1 \mathrm{n}-2}$ \\
\hline Number of Packets & $\mathrm{n}-2$ \\
\hline
\end{tabular}

Fig. 20. $k=1$.

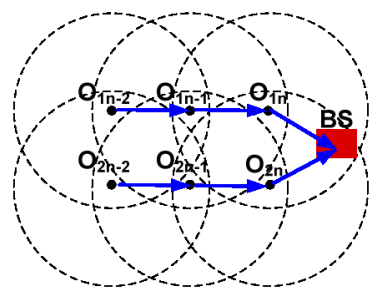

\begin{tabular}{|c|c|c|}
\hline Node & $\mathrm{O}_{1 \mathrm{n}-2}$ & $\mathrm{O}_{2 \mathrm{n}-2}$ \\
\hline Number of Packets & $\mathrm{n}-2$ & $\mathrm{n}-2$ \\
\hline
\end{tabular}

Fig. 21. $k=2$.
Case 4: $k=4$. For a 4-row grid topology network, the knowledge that we need to obtain the upper bound is given in Fig. 23. During the time period $x$, under the fair-access criterion, both $\mathrm{O}_{1 n}$ and $\mathrm{O}_{2 n}$ need to transmit at least $2 n$ frames to the BS. We have $b \geq 4 n T$. In order for $\mathrm{O}_{1 n}$ to receive $n-1$ frames from $\mathrm{O}_{1(n-1)}$ and receive $n$ frames from $\mathrm{O}_{3 n}, \mathrm{O}_{1 n}$ must listen to at least $2 n-1$ frames, during which time $\mathrm{O}_{2 n}$ cannot transmit (i.e., the BS must be idle). Similarly, $\mathrm{O}_{2 n}$ also needs to listen to at least $2 n-1$ frames, during which time $\mathrm{O}_{1 n}$ cannot transmit. But note that, when $\mathrm{O}_{1 n}$ receives frames, $\mathrm{O}_{2 n}$ can also receive frames. Furthermore, note that when $\mathrm{O}_{3(n-1)}$ transmits, $\mathrm{O}_{1 n}$ cannot transmit but $\mathrm{O}_{2 n}$ can. Likewise, when $\mathrm{O}_{1(n-2)}$ transmits, $\mathrm{O}_{1 n}$ cannot transmit but $\mathrm{O}_{2 n}$ can. Similarly, when $\mathrm{O}_{4(n-1)}$ transmits, $\mathrm{O}_{2 n}$ cannot transmit but $\mathrm{O}_{1 n}$ can. Likewise, when $\mathrm{O}_{2(n-2)}$ transmits, $\mathrm{O}_{2 n}$ cannot transmit but $\mathrm{O}_{1 n}$ can. Therefore, the total time in which neither $\mathrm{O}_{1 n}$ nor $\mathrm{O}_{2 n}$ can transmit is $y \geq(2 n-1) T$. Thus, we have $x=b+y \geq 4 n T+(2 n-1) T$. The upper bound is $U(4 n) \leq 4 n /(6 n-1)$. We also proved this upper bound can be achieved by a scheduling algorithm in our previous work [18].

Case 5: $k=5$. For a 5-row grid topology network, the knowledge that we need to obtain the upper bound is given in Fig. 24. Under fair-access criterion, during time period $x$,

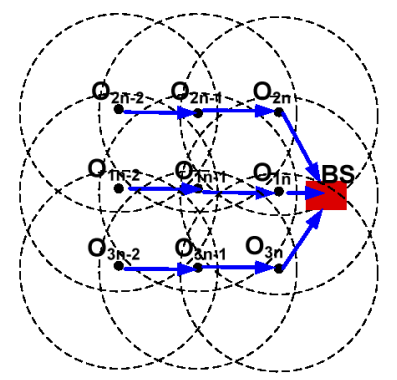

\begin{tabular}{|c|c|c|c|}
\hline Node & $\mathrm{O}_{1 \mathrm{n}-2}$ & $\mathrm{O}_{2 n-2}$ & $\mathrm{O}_{3 n-2}$ \\
\hline Number of Packets & $\mathrm{n}-2$ & $\mathrm{n}-2$ & $\mathrm{n}-2$ \\
\hline
\end{tabular}

Fig. 22. $k=3$. 


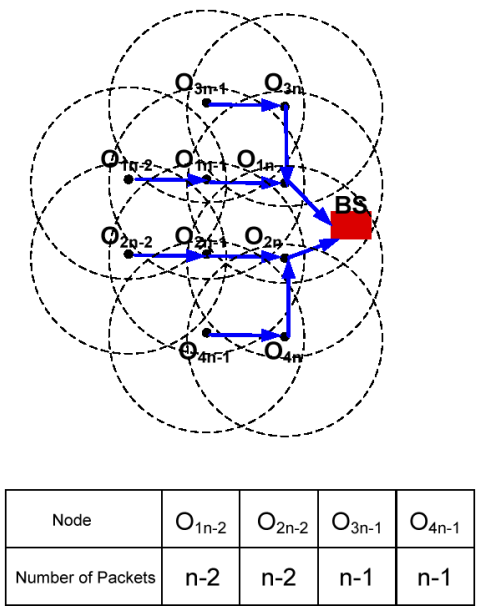

Fig. 23. $k=4$.

$\mathrm{O}_{1 n}$ needs to transmit at least $n$ frames to the BS. $\mathrm{O}_{2 n}$ and $\mathrm{O}_{3 n}$ each need to transmit at least $2 n$ frames to the BS. We have $b \geq 5 n T$. In order for $\mathrm{O}_{1 n}$ to receive $n-1$ frames from $\mathrm{O}_{1(n-1)}, \mathrm{O}_{1 n}$ must listen to at least $n-1$ frames, during which time both $\mathrm{O}_{2 n}$ and $\mathrm{O}_{3 n}$ cannot transmit (i.e., the BS must be idle). Except for node $\mathrm{O}_{1(n-1)}$ and for other nodes more than two hops away from the BS, when they transmit, there always exists a node from $\mathrm{O}_{1 n}, \mathrm{O}_{2 n}$, and $\mathrm{O}_{3 n}$ which can transmit. For example, when $\mathrm{O}_{2(n-1)}$ transmits, $\mathrm{O}_{1 n}$ and $\mathrm{O}_{2 n}$ cannot transmit but $\mathrm{O}_{3 n}$ can. Therefore, the total time when none of $\mathrm{O}_{1 n}, \mathrm{O}_{2 n}$, and $\mathrm{O}_{3 n}$ can transmit is $y \geq(n-1) T$. Thus, we have $x=b+y \geq 5 n T+(n-1) T$. The upper bound is $U(5 n) \leq 5 n /(6 n-1)$.

Case 6: $k$ is even and $k \geq 6$. For the case where $k$ is even and $k \geq 6$, the knowledge that we need to obtain the upper bound is given in Fig. 25. In other words, any complicated grid topology with an even number of rows can be simplified to Fig. 25. As mentioned above, $k$ can be denoted as $2 m$ in this case. During the time period $x$, under fairaccess criterion, both $\mathrm{O}_{1 n}$ and $\mathrm{O}_{2 n}$ need to transmit at least $m n$ frames to the BS. We have $b \geq 2 m n T$. In order for $\mathrm{O}_{1 n}$ to receive $n-1$ frames from $\mathrm{O}_{1(n-1)}$ and $(m-1) n$ frames from $\mathrm{O}_{3 n}, \mathrm{O}_{1 n}$ must listen to at least $m n-1$ frames, during which time $\mathrm{O}_{2 n}$ cannot transmit (i.e., the $\mathrm{BS}$ must be idle). Similarly, $\mathrm{O}_{2 n}$ also needs to listen to at least $m n-1$ frames, during which time $\mathrm{O}_{1 n}$ cannot transmit. But note that $\mathrm{O}_{1 n}$ and $\mathrm{O}_{2 n}$ can receive frames at the same time. Furthermore, when nodes which are three hops away from BS transmit,
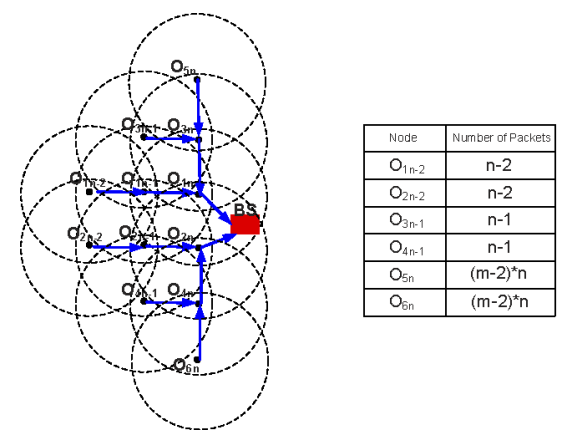

Fig. 25. $k$ is even and $k \geq 6$.

there always exists a node from $\mathrm{O}_{1 n}$ and $\mathrm{O}_{2 n}$ which can transmit. For example, when $\mathrm{O}_{5 n}$ transmits, $\mathrm{O}_{1 n}$ cannot transmit but $\mathrm{O}_{2 n}$ can. Therefore, the total time when neither $\mathrm{O}_{1 n}$ nor $\mathrm{O}_{2 n}$ can transmit is $y \geq(m n-1) T$. Thus, we have $x=b+y \geq 2 m n T+(m n-1) T$. The upper bound is $U(4 n) \leq 2 m n /(3 m n-1)$.

Case 7: $k$ is odd and $k \geq 7$. For the case where $k$ is odd and $k \geq 7$, the knowledge that we need to obtain the upper bound is given in Fig. 26. In other words, any complicated grid topology with an odd number of rows can be simplified to Fig. 26. As mentioned above, $k$ can be denoted as $2 m+1$ in this case. Under fair-access criterion, during time period $x, \mathrm{O}_{1 n}$ needs to transmit at least $n$ frames to the BS. $\mathrm{O}_{2 n}$ and $\mathrm{O}_{3 n}$ each need to transmit at least $m n$ frames to the BS. We have $b \geq(2 m+1) n T$. In order for $\mathrm{O}_{1 n}$ to receive $n-1$ frames from $\mathrm{O}_{1(n-1)}, \mathrm{O}_{1 n}$ must listen to at least $n-1$ frames, during which time both $\mathrm{O}_{2 n}$ and $\mathrm{O}_{3 n}$ cannot transmit (i.e., the BS must be idle). Except for node $\mathrm{O}_{1(n-1)}$, when other nodes with more than two hops away from BS transmit, there always exists a node from $\mathrm{O}_{1 n}, \mathrm{O}_{2 n}$, and $\mathrm{O}_{3 n}$ which can transmit. For example, when $\mathrm{O}_{6 n}$ transmits, $\mathrm{O}_{2 n}$ cannot transmit but $\mathrm{O}_{2 n}$ and $\mathrm{O}_{3 n}$ can. Therefore, the total time when none of $\mathrm{O}_{1 n}, \mathrm{O}_{2 n}$, and $\mathrm{O}_{3 n}$ can transmit is $y \geq(n-1) T$. Thus, we have $x=b+y \geq$ $(2 m+1) n T+(n-1) T$. The upper bound is $U(5 n) \leq(2 m+$ 1) $n /[(2 m+2) n-1]$. Note that upper bounds given in Cases 5, 6, 7 are not necessarily tight.

\subsection{Acoustic-Based Underwater Sensor Network}

In this section, we discuss the upper bounds on network utilization in multiline networks. Theorem 11 derives the upper bound based on the conclusion of Theorem 7 .

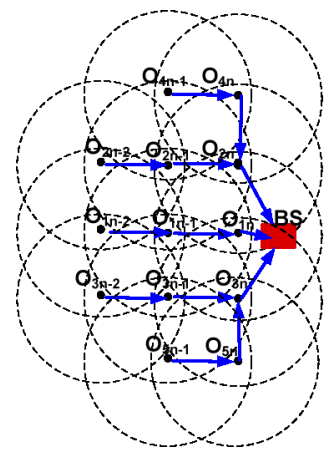

Fig. 24. $k=5$.

\begin{tabular}{|c|c|}
\hline Node & Number of Packets \\
\hline $\mathrm{O}_{1 \mathrm{n}-2}$ & $\mathrm{n}-2$ \\
\hline $\mathrm{O}_{2 n-2}$ & $\mathrm{n}-2$ \\
\hline $\mathrm{O}_{3 n-2}$ & $\mathrm{n}-2$ \\
\hline $\mathrm{O}_{4 n-1}$ & $\mathrm{n}-1$ \\
\hline $\mathrm{O}_{5 n-1}$ & $\mathrm{n}-1$ \\
\hline
\end{tabular}

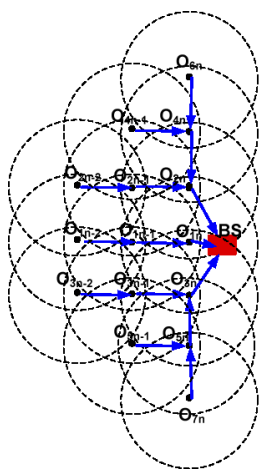

Fig. 26. $k$ is odd and $k \geq 7$. 


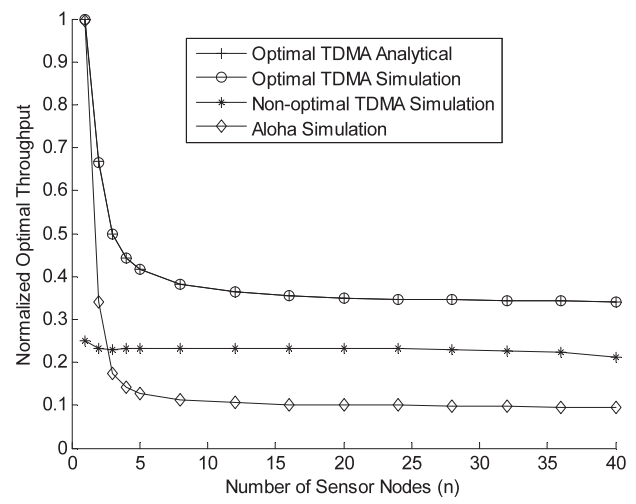

Fig. 27. Simulation results for the linear topology.

Theorem 11. Let $n$ denote the total number of nodes in the network and $M$ represent the branch with the maximum number of nodes. The number of nodes in branch $M$ is denoted as $n_{M}$. The lower bound on the cycle time is $\max \left(\left(n_{M}-\right.\right.$ 1) $(3 T-2 \tau)+2 \tau, n)$.

Proof. First, under the constraint of fair access, the base station is required to receive at least one frame from each node in the circle. Thus, $n$ is a lower bound in any network. Furthermore, according to the optimal fair scheduling for linear topology, the minimum cycle time for branch $M$ is $\left(n_{M}-1\right)(3 T-2 \tau)$. Thus, the lower bound on the cycle time is $\max \left(\left(n_{M}-1\right)(3 T-2 \tau)+2 \tau, n\right)$.

\section{Simulation Results}

In this section, we provide simulation results on throughput for linear topology and grid topology. Simulations are conducted with discrete event simulation using Java. In our simulations, the transmission range of each node is just one hop and the interference range is less than two hops. In other words, only neighboring nodes have overlapping transmission ranges. Other characteristics, such as variable propagation delay, frequency dependent path loss, and fading noise are not considered in this simulations. Fig. 27 shows the normalized utilization versus the number of nodes for the linear topology. As illustrated in Fig. 27, for the optimal fair TDMA scheduling mentioned in Section 4, the analytical results exactly match the simulation results. For showing optimal TDMA scheduling indeed has better performance than other scheduling algorithms, a specific TDMA and Aloha are simulated. Here, we briefly specify the TDMA scheduling. In the specific nonoptimal TDMA scheduling, a node with hop-count $h$ is assigned time slots of

1. $4 i+1$ to send available frames if $h \bmod 4$ is 1 ,

2. $4 i+2$ to send available frames if $h \bmod 4$ is 2 ,

3. $4 i+3$ to send available frames if $h \bmod 4$ is 3 , and

4. $4 i+4$ to send available frames if $h \bmod 4$ is 0 , where $i=0,1,2, \ldots \ldots$.. From Fig. 27, we can observe that, although this specific TDMA scheduling is not optimal, it still has better throughput performance than Aloha. In order to show the upper bound of throughput on general $k \times n$ grid topology, we simulate an Aloha protocol for a specific grid topology network where $k$ is 6 . As illustrated in Fig. 28, the optimal analytical bound is far better than

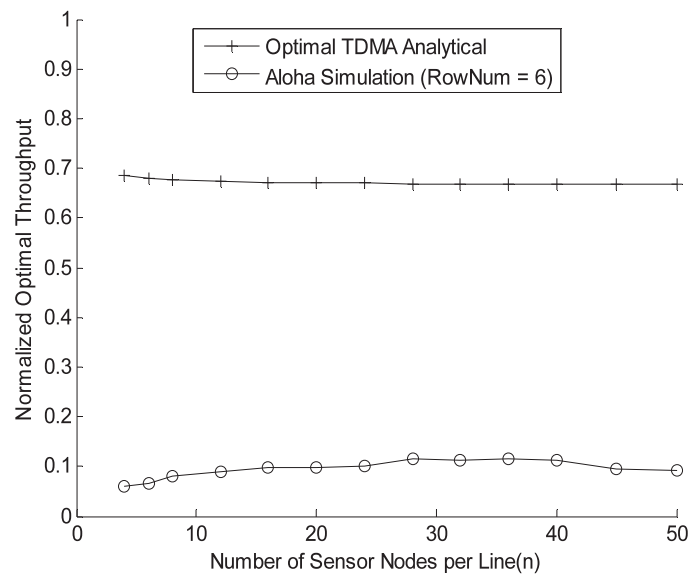

Fig. 28. Simulation results for grid topology.

simulation result of Aloha protocol. Furthermore, we explore the impact of the size of grid network on throughput by simulations. As illustrated in Fig. 29, when the size of grid network becomes larger, the throughput becomes small. That is because the nodes connecting to the BS will stay the same no matter how large the grid network is. Therefore, large network causes more traffic collisions and lead to low throughput.

\section{Conclusion AND Future Work}

In this paper, we explored fundamental limits for sustainable loads, utilization, and delays in specific multihop sensor network topologies for both wireless sensor networks and underwater acoustic sensor networks. We derived upper bounds on network utilization and lower bounds for minimum sample time in fixed linear and multirow grid topologies under the fair-access criterion. This fair-access criterion ensures that the data of all sensors are equally capable of reaching the base station. We proved that under some conditions/assumptions, these bounds are achievable and therefore optimal. From the limitation on the sustainable traffic loads derived, one can determine a lower bound for the sampling interval for such networks. The significance of these limits is that these bounds are independent of the selection of MAC protocols under both single-channel and half-duplex radios. Thus,

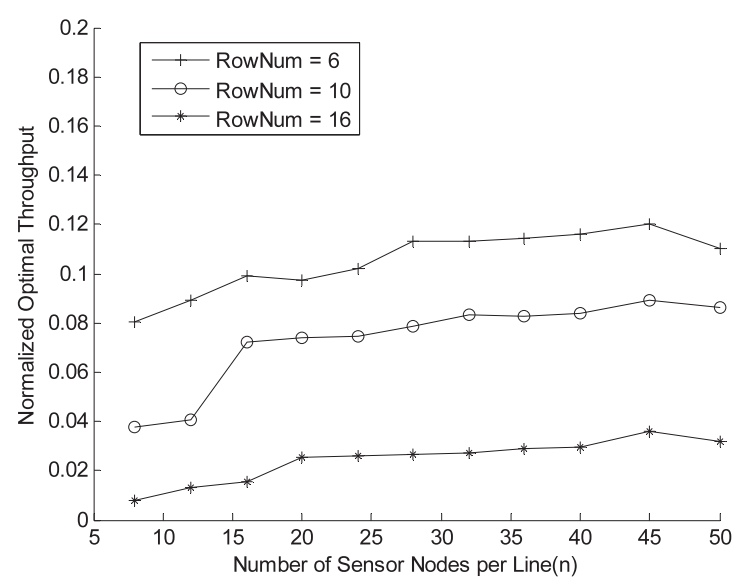

Fig. 29. Impact of row number on Aloha simulation. 
the performance bounds for specific implementations of such network topologies can be explicitly determined to ensure the proposed networks are capable of satisfying the networks' specified utilization and delay requirements. Further, a self-clocking implementation was described that achieves the utilization bounds.

MAC protocols in WLANs/WPANs such as 802.3 (Ethernet), 802.11 (WiFi), 802.15.1 (Bluetooth), 802.15.3, and 802.15.4 (ZigBee) are contention-based (such as CSMA/CD, CSMA/CA, etc.), contention-free (such as polling), or hybrid. Under a single-channel and a half-duplex radio, our bounds hold for all of these MAC protocols, where a particular optimal TDMA can achieve the tight bound. For contention-based MAC, the bound could not be achieved due to collisions involved (please refer to the detail of the proofs of tight bounds).

Note that even though we assume acknowledgments are implicit, our bounds still apply when explicit acknowledgments are used, but they are no longer tight bounds. Obtaining tight bounds for explicit acknowledgments are our future work.

As other future work, we will investigate whether optimal schedules exist for irregular topologies and various routing schemes under the fair-access constraint. For underwater sensor networks, further analysis for $\tau>T / 2$ is necessary. Moreover, we will further loosen the assumptions in this paper and explore how to apply our analysis method to other networks with different constraints. For example, instead of assuming that the spacing and propagation delays are fixed and equal, we assume there are always spacing and propagation delay errors existing in wireless sensor network. We will also explore whether our analysis method can be extended to other network types where both sides of the base station could have sensor nodes or the communication range could be larger such that two-hop or even more hops neighbors can hear messages.

\section{ACKNOWLEDGMENTS}

This work is supported in part by the US National Science Foundation under grant numbers CNS-0737325, CNS0716211, CCF-0829827, and CNS-1059265. Ding-Zhu Du's work was supported in part by MEST, Korea under WCU (R33-2008-000-10044-0), by a KOSEF grant funded by the Korea government (MEST) (No. R01-2007-000-11203-0), by a KRF Grant funded by (KRF-2008-314-D00354), and by MKE, Korea under ITRC IITA-2009-(C1090-0902-0046) and IITA2009-(C1090-0902-0007).

\section{REFERENCES}

[1] B. Benson, G. Chang, D. Manov, B. Graham, and R. Kastner, "Design of a Low-Cost Acoustic Modem for Moored Oceanographic Applications," Proc. First ACM Int'l Workshop Underwater Networks (WUWNet'06), Sept. 2006.

[2] R. Ramaswami and K.K. Parhi, "Distributed Scheduling of Broadcasts in a Radio Network," Proc. IEEE INFOCOM, 1989.

[3] S. Ramanathan, "A Unified Framework and Algorithm for Channel Assignment in Wireless Network," Wireless Networks, vol. 5, no. 2, pp. 81-94, 1999.

[4] V. Rajendran, K. Obraczka, and J.J. Garcia-Luna-Aceves, "EnergyEfficient, Collision-Free Medium Access Control for Wireless Sensor Networks," Proc. ACM First Int'l Conf. Embedded Networked Sensor Systems (Sensys '03), pp. 181-192, Nov. 2003.
[5] J. Gibson, G.G. Xie, and Y. Xiao, "Performance Limits of FairAccess in Sensor Networks with Linear and Selected Grid Topologies," Proc. IEEE GlobeCom, pp. 688-693, 2007.

[6] G. Xie, J. Gibson, and Y. Xiao, "Analyzing the Performance of Multihop Underwater Acoustic Sensor Networks," Proc. MTS/ IEEE OCEANS Conf., 2007.

[7] F. Hu, Y. Malkawi, S. Kumar, and Y. Xiao, "Vertical and Horizontal Synchronization Services with Outlier Detection in Underwater Sensor Networks," Wireless Comm. and Mobile Computing J., vol. 8, no. 9, pp. 1165-1181, Nov. 2008, doi:10.1002/wcm.559.

[8] F. Hu, P. Tilghman, Y. Malkawi, and Y. Xiao, "A Prototypeunderwater Acoustic Sensor Network Platform with Topology-Aware MAC Scheme," Int'l J. Sensor Networks, vol. 2, nos. 5/6, pp. 386398, 2007.

[9] Y. Zhang, Y. Xiao, M. Chen, P. Bahri, and M. Kamboj, "Medium Access Control Layer for Underwater Sensor Networks," Underwater Acoustic Sensor Networks, Auerbach Publications, 2009.

[10] Y. Huang, W. Liang, H.-B. Yu, and Y. Xiao, "Target Tracking Based on a Distributed Particle Filter in Underwater Sensor Networks," Wireless Comm. and Mobile Computing, Special Issue on Underwater Sensor Networks: Architectures and Protocols, vol. 8, no. 8, pp. 1011-1022, Oct. 2008, doi:10.1002/wcm.656.

[11] L. Liu, Y. Xiao, and J. Zhang, "Effect of Node Movement to Time Synchronization of Underwater Wireless Sensor Network," Proc. IEEE Int'l Conf. Comm. (ICC), 2009.

[12] S. Gandham, Y. Zhang, and Q. Huang, "Distributed Minimal Time Convergecast Scheduling in Wireless Sensor Networks," Proc. IEEE 26th Int'l Conf. Distributed Computing Systems (ICDCS), 2006.

[13] J. Wang Hongsik Choi and E.A. Hughes, "Scheduling on Sensor Hybrid Network," Proc. IEEE 14th Int'l Conf. Computer Comm. and Networks (ICCCN), 2005.

[14] S.C. Ergen and P. Varaiya, "TDMA Scheduling Algorithms for Wireless Sensor Networks," Wireless Networks, vol. 16, pp. 985-997, 2010.

[15] S.C. Ergen and P. Varaiya, "Power Efficient and Delay Aware Medium Access Protocol for Sensor Networks," IEEE Trans. Mobile Computing,", vol. 5, no. 7, pp. 920-930, July 2007.

[16] C. Florens, M. Franceschetti, and R.J. McEliece, "Lower Bounds on Data Collection Time in Sensory Networks," IEEE J. Selected Areas in Comm., vol. 22, no. 6, pp. 1110-1120, Aug. 2004.

[17] C. Florens and R. McEliece, "Packets Distribution Algorithms for Sensor Networks," Proc. IEEE INFOCOM, 2003.

[18] M. Peng and Y. Xiao, "Upper Bound on Network Utilization under Fair-Access in Multi-Hop Wireless Grid Sensor Networks with 3-4,Rows," Proc. Fifth Int'l Conf. Mobile Ad-Hoc and Sensor Networks (MSN '09), pp. 244-251, 2009.

[19] Y. Xiao and J. Rosdahl, "Throughput Analysis for IEEE 802.11a Higher Data Rates," Proc. IEEE 802.11 Plenary Meeting, Mar. 2002.

[20] Y. Xiao, "Throughput and Delay Limits of IEEE 802.11," IEEE Comm. Letters, vol. 6, no. 8, pp. 355-357, Aug. 2002.

[21] G.G. Xie and J. Gibson, "A Networking Protocol for Underwater Acoustic Networks," Technical Report TR-CS-00-02, Dept. of Computer Science, Naval Postgraduate School, Dec. 2000.

[22] D. Pompili, T. Melodia, and I.F. Akyildiz, "A Distributed CDMA Medium Access Control for Underwater Acoustic Sensor Networks," Proc. Mediterranean Ad Hoc Networking Workshop (MedHoc-Net), 2007.

[23] L.F.M. Vieira, J. Kong, U. Lee, and M. Gerla, "Analysis of ALOHA Protocols for Underwater Acoustic Sensor Networks," Proc. First ACM Int'l Workshop UnderWater Networks (WUWNet), 2006.

[24] N. Chirdchoo, W.S. Soh, and K.C. Chua, "ALOHA-Based MAC Protocols with Collision Avoidance for Underwater Acoustic Networks," Proc. IEEE INFOCOM, 2007.

[25] M. Molins and M. Stojanovic, "Slotted FAMA a MAC Protocol for Underwater Acoustic Networks," Proc. MTS/IEEE Conf. and Exhibition for Ocean Eng., Sept. 2006.

[26] A.A. Syed, W. Ye, and J. Heidemann, "T-Lohi: A New Class of MAC Protocols for Underwater Acoustic Sensor Networks," Technical Report ISI-TR-638, Univ. of Southern California, Information Sciences Inst., Apr. 2007.

[27] F. Salva-Garau and M. Stojanovic, "Multi-Cluster Protocol for Ad Hoc Mobile Underwater Acoustic Networks," Proc. MTS/IEEE OCEANS, Sept. 2003. 


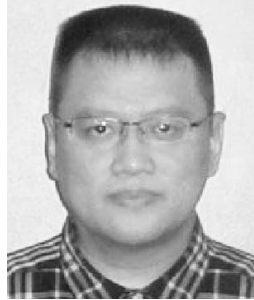

Yang Xiao worked in industry as a Medium Access Control (MAC) architect involved in the IEEE 802.11 standard enhancement work before he joined academia. He is currently with Department of Computer Science (with tenure) at The University of Alabama. He was a voting member of the IEEE 802.11 Working Group from 2001 to 2004. His research areas are in security and communications/networks. He has published more than 200 refereed journal papers and more than 200 refereed conference papers and book chapters related to these research areas. He currently serves as the editor-in-chief for the International Journal of Security and Networks (IJSN) and the International Journal of Sensor Networks (IJSNet). He was the founding editor-in-chief for the International Journal of Telemedicine and Applications (IJTA) (2007-2009). He is a senior member of the IEEE.

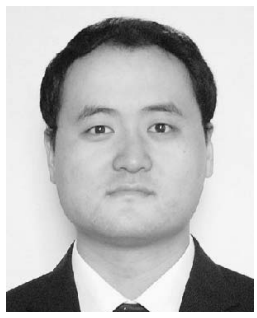

Miao Peng received the BS degree in applied mathematics from the Dalian University of Technology, China, in 2004 and the MS degree in mathematical statistics from Jilin University, Changchun, China, in 2007. He is currently working toward the $\mathrm{PhD}$ degree in computer science with The University of Alabama, Tuscaloosa. His research interests include wireless sensor networks, wireless network security, and energy-efficient wireless networks. In particular, he is interested in mathematical modeling in wireless and sensor networks.

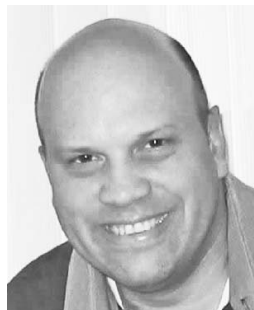

John Gibson received the BA degree from Point Loma Nazarene College in 1977, the MS degree from the US Naval Postgraduate School (NPS) in 1990, and the second MS degree from NPS in 2000. He is currently working toward the $\mathrm{PhD}$ degree in software engineering. From 1981-1995, he was a communications and computer systems officer (various USAF assignments). From 1995-2000, he taught Joint C4I at NPS. Since 2001, he has been both a research associate and lecturer in the NPS Department of Computer Science.

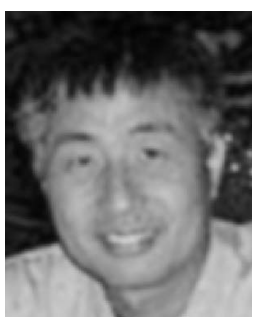

Geoffrey G. Xie received the BS degree in computer science from Fudan University, China and the PhD degree in computer sciences from the University of Texas, Austin. He is a professor in the Computer Science Department at the US Naval Postgraduate School. He was a visiting scientist in the School of Computer Science at Carnegie Mellon University from 2003 to 2004 and recently visited the Computer Laboratory of the University of Cambridge, United Kingdom. He has published more than 60 articles in various areas of networking. He was an editor of the Computer Networks journal from 2001 to 2004 . He cochaired the ACM SIGCOMM Internet Network Management Workshop in 2007 and is currently a member of the workshop's steering committee. His current research interests include network analysis, routing design and theories, underwater acoustic networks, and abstraction driven design and analysis of enterprise networks.

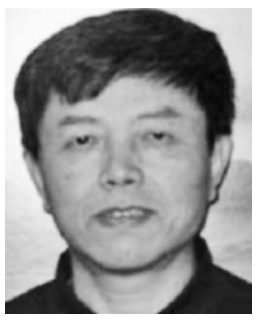

Ding-Zhu Du received the MS degree in 1982 from the Institute of Applied Mathematics, Chinese Academy of Sciences, and the PhD degree in 1985 from the University of California at Santa Barbara. He worked in the Mathematical Sciences Research Institute, Berkeley, California, in 1985-1986, at the Massachusetts Institute of Technology from 1986-1987, and at Princeton University from 1990-1991. He was an associate-professor/professor in the Department of Computer Science and Engineering, University of Minnesota, from 1991-2005, a professor at the City University of Hong Kong from 19981999, a research professor at the Institute of Applied Mathematics, Chinese Academy of Sciences, from 1987-2002, and a program director at the US National Science Foundation (NSF) from 2002-2005. Currently, he is a professor in the Department of Computer Science, University of Texas at Dallas, and a WCU professor at Korea University. His research interests include the design and analysis of algorithms for combinatorial optimization problems in communication networks and bioinformatics. He has published more than 160 journal papers and 10 books. He is the editor-in-chief of the Journal of Combinatorial Optimization and Discrete Mathematics, Algorithms and Applications. $\mathrm{He}$ is also on the editorial boards of more than 15 journals.

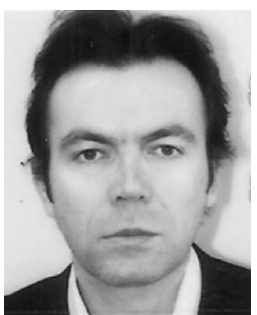

Athanasios V. Vasilakos is currently a visiting professor at the National Technical University of Athens, Greece. He has authored or coauthored more than 200 technical papers in major international journals and conferences. $\mathrm{He}$ is an author/coauthor of five books and 20 book chapters in the area of communications. He has served as a general chair or technical program committee chair for many international conferences. He served or is serving as an editor and/ or guest editor for many technical journals, such as the IEEE Transactions on Network and Services Management, the IEEE Transactions on Systems, Man, and Cybernetics-Part B: Cybernetics, the IEEE Transactions on Information Technology in Biomedicine, ACM Transactions on Autonomous and Adaptive Systems, IEEE JSAC special issues in May 2009, January 2011, March 2011, IEEE Communications Magazine, ACM/Springer Wireless Networks (WINET), and ACM/ Springer Mobile Networks and Applications (MONET). He is the founding editor-in-chief of the International Journal of Adaptive and Autonomous Communications Systems (IJAACS, http://www.inderscience.com/ ijaacs) and the International Journal of Arts and Technology (IJART, http://www.inderscience.com/ijart). He is the general chair of the Council of Computing of the European Alliances for Innovation. He is a senior member of the IEEE.

$\triangleright$ For more information on this or any other computing topic, please visit our Digital Library at www.computer.org/publications/dlib. 